\title{
Development of a computerized assessment tool for hand-arm function after stroke- test-retest reliability and convergent validity
}

\author{
By \\ Zoya Imran \\ A thesis submitted to the Faculty of Graduate Studies of \\ The University of Manitoba \\ In partial fulfillment of the requirements of the degree of
}

\section{MASTER OF SCIENCE}

College of Rehabilitation Sciences

Rady Faculty of Health Sciences

University of Manitoba

Winnipeg

Copyright (C) 2016 by Zoya Imran 


\begin{abstract}
Many performance-based assessment tools are available to measure upper extremity function. Most define hand function by the time taken to complete a task. A new computer gamebased hand-arm function evaluation (GHA) tool has been developed to quantify fine and gross object manipulations skills. Objective of this study was to evaluate the test-retest reliability and convergent validity of the GHA assessment tool. Thirty stroke clients with the mean age of 68.41 years were recruited. Test-retest reliability was assessed by Intraclass correlation coefficient (ICCs) and t-test. Convergent validity between GHA and the Wolf Motor Function Test (WMFT) was determined using the Pearson's correlation coefficient. Most of the GHA outcome measures had moderate to high ICC (0.5-0.9). With scant exceptions, low correlations were observed between GHA measures and WMFT score. The ICC values reflected the complexity of the tasks, more complex task showed lower ICCs values. The WMFT measures the time taken to complete a task whereas GHA measures the movement quality and accuracy. Thus not measuring similar characteristics of a movement might be the reason for low correlations between GHA and WMFT.
\end{abstract}




\section{Acknowledgements}

I am for always thankful to my advisor Dr. Tony Szturm and my committee members Dr. Sepideh Pooyania and Dr. Barbara Shay. I express my highest regards and gratitude towards them for believing in me and offering me this fascinating project. I am equally thankful to them

for their excellent support, motivation and all the hard work they have brought into this project.

I am truly grateful to all the study participants for putting their faith in me and showing all the enthusiasm and participation in this project. Without them, this achievement wouldn't have been possible.

I am very thankful to the Riverview health center for providing us their annual research grant. I am also much grateful to the PT and OT staff at the Riverview health center for their assistance, without which this project wouldn't have been in a position to come this far.

I would also like to extend my warmest appreciations to my friends and fellow graduate students for their unceasing support and being so wonderful.

I am further grateful to the University of Manitoba for providing me the Manitoba graduate scholarship, which has helped me focus on my studies and complete them in time.

Lastly, I am immensely thankful to my family for all of their support and the efforts they have put into making me a better person. 


\section{Dedication}

This thesis work is dedicated to my parents- M. Imran and Farhana Saghir

Zoya Imran 


\section{TABLE OF CONTENTS}

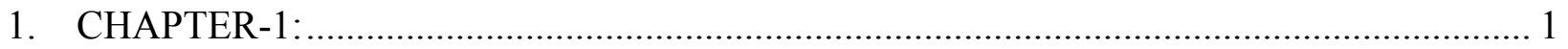

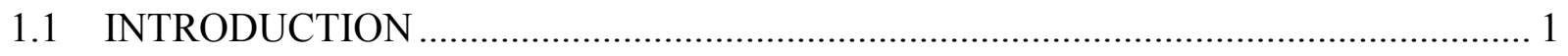

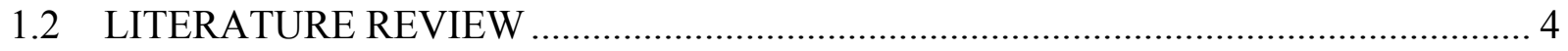

1.2.1 CONSTRAINED INDUCED MOVEMENT THERAPY (CIMT) ........................... 4

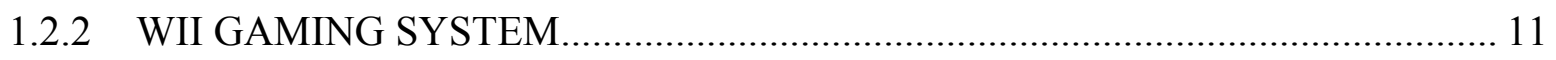

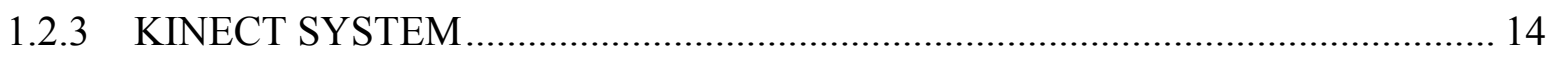

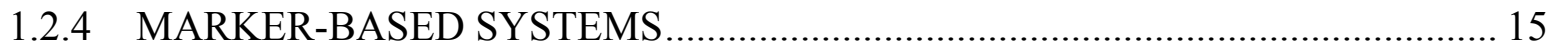

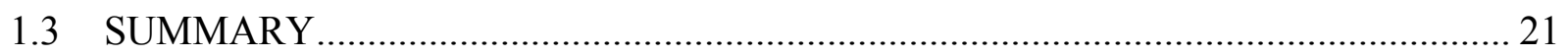

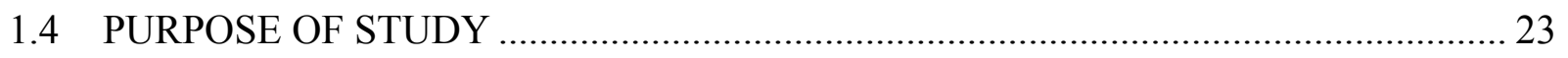

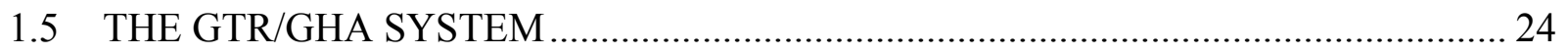

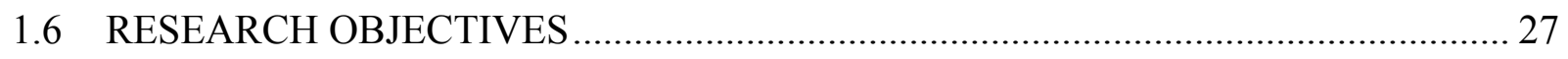

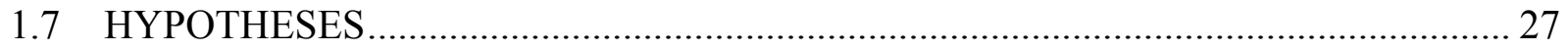

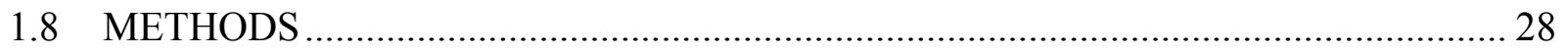

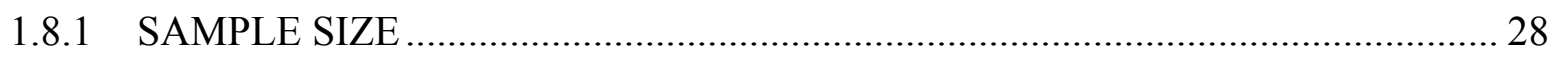

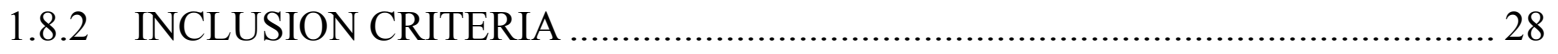

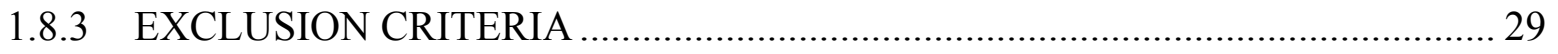

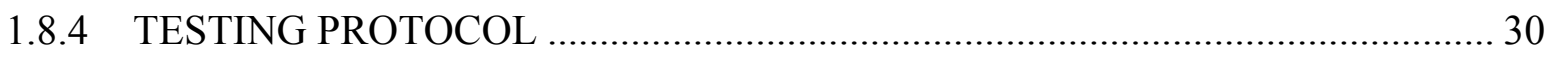




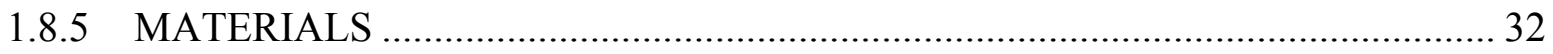

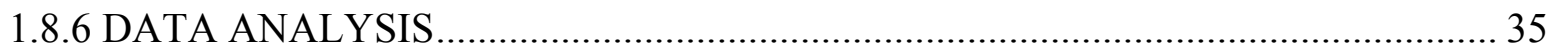

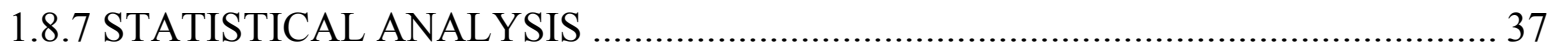

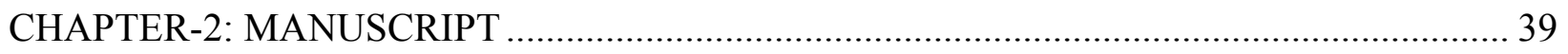

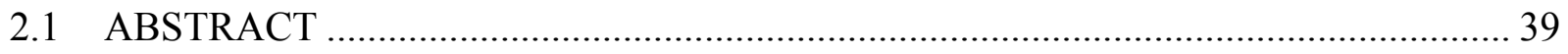

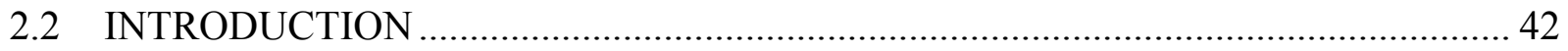

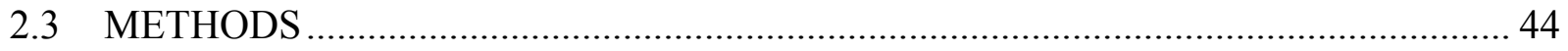

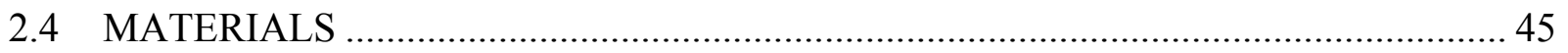

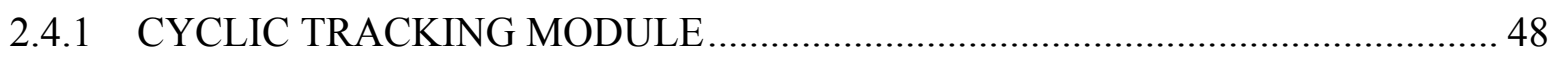

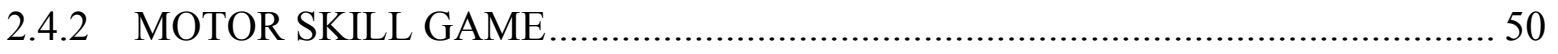

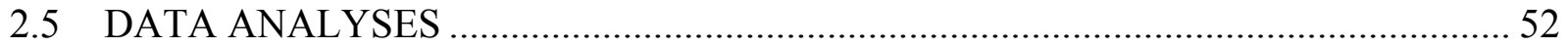

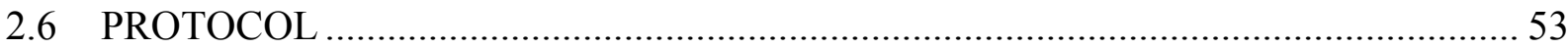

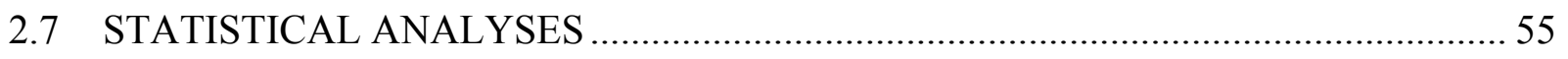

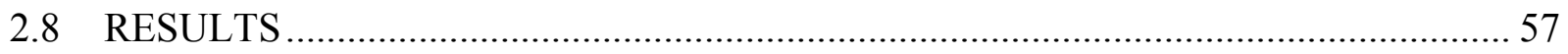

2.8.1 RAW DATA OF THE GHA ASSESSMENT OUTCOMES AND THEIR KEY

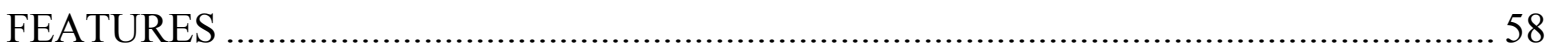

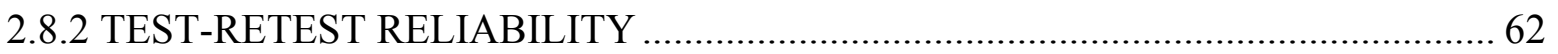

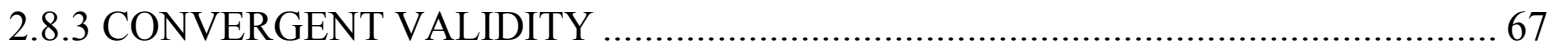

2.8.4 CORRELATION OF TASKS WITHIN STROKE GROUP …………………....... 68

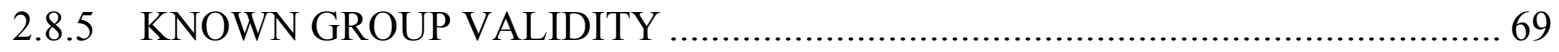




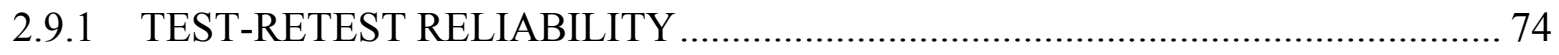

2.9.2 CONVERGENT VALIDITY AND KNOWN GROUP VALIDITY ...................... 79

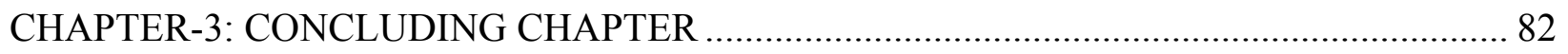

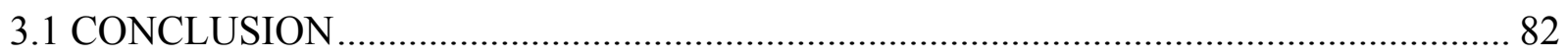

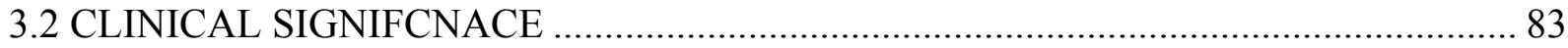

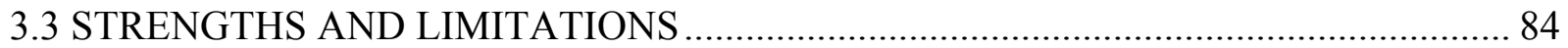

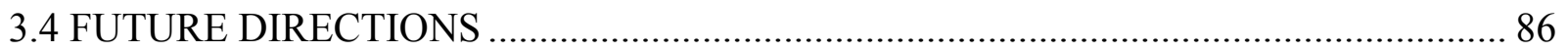

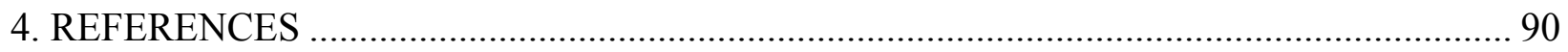




\section{LIST OF TABLES}

TABLE 1- OBJECTS MANIPULATED AND THEIR RESPECTIVE MOVEMENTS................................ 47

TABLE 2-CLINICAL AND DEMOGRAPHIC CHARACTERISTICS OF STUDY PARTICIPANTS................... 57

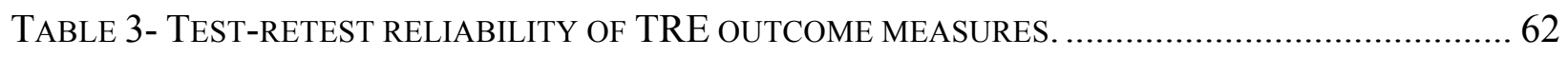

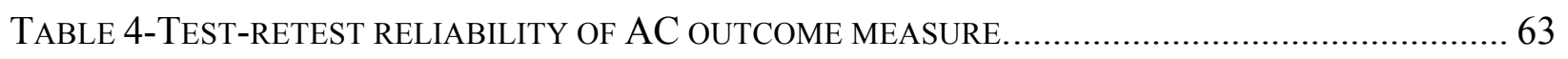

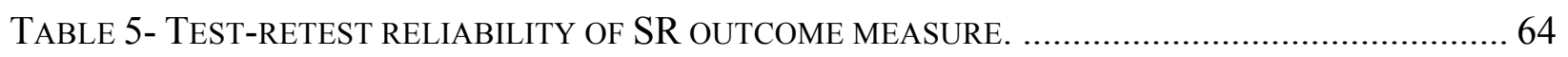

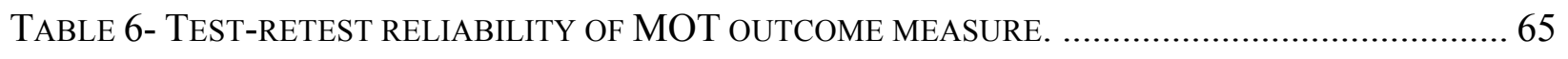

TABLE 7- TEST-RETEST RELIABILITY OF MV OUTCOME MEASURE.......................................... 66

TABLE 8- BETWEEN GROUP STATISTICAL ANALYSES OF TRE............................................ 70

TABLE 9-BETWEEN GROUP STATISTICAL ANALYSES OF MOT ............................................... 71

TABLE 10- BETWEEN GROUP STATISTICAL ANALYSES OF MV ….............................................. 72 


\section{LIST OF FIGURES}

FIGURE 1-THE RAW DATA OF CYCLIC TRACKING TASK........................................................ 36

FIGURE 2-THE RAW DATA OF MOTOR SKILL GAME TASK................................................. 37

FIGURE 3- CYCLIC TRACKING TASK, AND ITS COMPONENTS................................................... 49

FIGURE 4- MOTOR SKILL GAME TASK AND ITS COMPONENTS.................................................. 51

FIGURE 5- USER MOTION TRAJECTORIES OF CYCLIC TRACKING TASKS _.................................... 59

FIGURE 6- USER MOTION TRAJECTORIES OF MOTOR SKILL GAME TASKS...................................... 61

FIGURE 7- BETWEEN-GROUP COMPARISONS OF TRE. ….................................................... 70

FIGURE 8- BETWEEN GROUP COMPARISONS OF MOT. ......................................................... 71

FIGURE 9-BETWEEN GROUP COMPARISONS OF MV ......................................................... 72 


\section{Development of a Computerized assessment tool for hand-arm function after stroke: test- retest reliability and convergent validity}

\section{CHAPTER-1}

\subsection{INTRODUCTION}

Stroke is a major leading cause of disability in particular due to hemiparesis of upper and lower extremities. The annual burden caused by stroke is almost about $\$ 65.5$ billion, which makes the rehabilitation post stroke an important issue (1). One-fourth of the people who suffered a stroke have chronic dysfunctions, affecting their ability to stay independent in their daily living activities. Though there are motor dysfunctions after stroke both in the upper as well as in the lower extremity, upper extremity rehabilitation has been given comparatively less consideration in the acute stage setup (2).

Manual dexterity involving handling and manipulating objects with the fingers and hand is necessary for nearly all daily life activities, such as dressing, grooming, eating, use of utensils and implements, and participation in play, hobbies, and chores. These activities require manipulation of objects with a broad range of physical properties (size, shape, weight, inertia, location of mass center), and often require a high degree of precision, where small deviations in timing or end point positioning/orientation of the object leads to complete disruption of the performance. Thus, it is critical for the patient to gain the dexterity skills post stroke to decrease the dependency, prevent non-use sequelae, and ease the activities of daily living.

The Constrained Induced Movement Therapy (CIMT) forms the core basis of motor rehabilitation post stroke (3). Motor recovery post stroke is a tedious and time-consuming process, not many can afford regular physical therapy due to reasons such as money, transportation, etc. Furthermore, adherence to physical exercises decreases even more as the exercises become boring and monotonous after some time (4). 


\section{Development of a Computerized assessment tool for hand-arm function after stroke: test- retest reliability and convergent validity}

To overcome these difficulties, and make exercises more engaging and fun, researchers have been coupling them with digital media, or in simple words, computer games. Setups like Nintendo, Wii and marker-based movement tracking systems have been tested in rehabilitation setups and have given hope to better participant engagement than traditional therapies. However, there are still many perspectives, which have to be considered before these programs can be chosen over traditional ones in terms of better recovery. One major problem with these setups is that they are still not feasible for home transition and require one to one guidance.

With respect to better outcomes, how good a therapy program is, is measured by the results obtained by an outcome measure. Outcome measures developed so far are either time based or counting the number of objects moved, which are limited to pegs, coins, cards, etc. moved. As these outcomes do not tell us about the movement efficiency, accuracy and movement path, it is necessary to have a measure, which can be more accurate and objective.

To overcome all the above-stated problems in a therapy program as well in assessment, Szturm et al. have developed an innovative computer Game-based Tele-Rehabilitation platform (GTR) for the training of finger hand function with an embedded assessment program, been called the game based hand-arm assessment (GHA). The GTR couples exercise with interactive computer games. Using the task-oriented approach a variety of manipulation tasks with objects of daily life differing in sizes, shapes, weights, gravity assisted or eliminated and functional demands can be used to practice many gross or fine motor skills and importantly while playing computer games. With this tool, a personalized training program can be built, which could be tailored for each according to his or her needs. 
Development of a Computerized assessment tool for hand-arm function after stroke: testretest reliability and convergent validity

The GHA is a tele-monitoring application tool that evaluates finger hand function. The tele-monitoring module automatically logs clients' task performances. Data analysis methods have been developed to quantify movement quality during standardized, functional object manipulation tasks by the use of computer controlled game activities. These objective electronic outcomes can then be used as outcome measures to track changes as a function of intensity, volume of practice, and tolerance. 


\section{Development of a Computerized assessment tool for hand-arm function after stroke: test- retest reliability and convergent validity}

\subsection{LITERATURE REVIEW}

\subsubsection{Constrained induced movement therapy (CIMT)}

More than $50 \%$ of stroke survivors report persistent hemiparesis resulting in impaired fine and gross motor skills of the upper extremity (5), (6). Various studies have shown that early care, high intensity and functional involvement of the movements practiced is directly proportional to a better recovery (7), (8). Unfortunately, there is a natural tendency for stroke clients not to use the affected arm and hand, post stroke, and rather try to do almost every functional activity with their less affected upper extremity (1). This is known as the non-use phenomenon. This phenomenon further decreases functional ability of the affected arm and hand. CIMT tries to inhibit the non-use phenomenon by restraining the non-affected arm and forcing the affected one to perform all the functional activities with high intensity for many consecutive days (3).

CIMT involves forced use of the affected upper limb in various motor tasks for a period of 2-12 weeks. The less affected arm is placed in a mitt for 4-6 hours per day and the other arm is forced to perform various physical activities of daily living such as, lifting objects, opening doors, grooming, etc., and repeat them. Movements are practiced quite vigorously, and involve the shoulder, elbow, forearm wrist and hand. However, not much is done for the fine manipulation of finger and hand. As the unaffected arm is tied, bilateral activities cannot be practiced and supervision is required at all time during the sessions.

A number of studies have evaluated the effects of CIMT in patients post stroke. Wolf et al did a randomized multisite clinical trial to compare the effects of a 2-week CIMT program versus usual rehabilitation therapies on improvement in upper extremity motor functions among stroke clients (3). Two hundred twenty-two sub-acute stroke clients, having mild to moderate 


\section{Development of a Computerized assessment tool for hand-arm function after stroke: test- retest reliability and convergent validity}

motor dysfunctions, were randomly assigned to the control and experimental group. The control group therapy ranged from no treatment to basic rehabilitation therapies involving stretching, strengthening and range of motion exercises, whereas CIMT group practiced repetitive tasks involving functional activities like eating, lifting objects, etc., for 6 hours a day. The duration of treatment, if given to control group is not explained in detail, but it would have been less than CIMT group as there were participants in the control group who did not get any treatment at all. Functional outcomes in both the groups were measured with WMFT and the Motor Activity Log (MAL). The WMFT quantitatively measures upper limb motor ability by recording the time taken for completing 15 functional tasks. 120 seconds are given to complete each task, the lesser the time taken, the better are functions. Two strength-based tasks are also included which measure pinch and grip strength by a dynamometer (8), (9). The MAL on the other hand is a semi-structured interview to assess upper limb motor functions. Participants are asked to rate 30 tasks of daily living on the basis of quality of movement and amount of movement on ordinal scales of $0-5$, with zero being the least activity or quality and 5 being the highest. Scores are based on an average of all tasks on a scale of 0-5, i.e. a mean MAL score is calculated for both scales by adding the rating scores for each scale and dividing by the number of items asked (10). At the end of the 2 week program, CIMT group showed clinically significant improvements in upper-extremity function compared to the control group as the mean time taken to complete WMFT decreased from $19.3 \mathrm{~s}$ to $10.8 \mathrm{~s}$ versus $24.0 \mathrm{~s}$ to $22.4 \mathrm{~s}$, grip strength increased from 7.53 $\mathrm{kg}$ to $9.51 \mathrm{~kg}$ versus $7.23 \mathrm{~kg}$ to $7.91 \mathrm{~kg}$, amount of use in the Motor Activity Log increased from 1.21 to 2.24 versus 1.15 to 1.37 . Furthermore, improvements were seen from baseline to $12-$ month follow-up within the group on all upper extremity outcomes as the mean time for WMFT decreased from $19.3 \mathrm{~s}$ to $9.3 \mathrm{~s}$ versus 24.0 to $17.7 \mathrm{~s}$ making the between-group difference $34 \%$ 


\section{Development of a Computerized assessment tool for hand-arm function after stroke: test- retest reliability and convergent validity}

$(\mathrm{P}<0.001)$. The MAL increased from 1.21 to 2.13 versus 1.28 to 1.66 , showing between group differences of $0.43(\mathrm{P}<0.001)$. The Stroke Impact Scale (SIS) showed a decrease of 19.5 in selfperceived hand functional difficulty whereas the control group decreased from 10.1 having a within group difference of $9.42(\mathrm{P}-0.05)$. Although there was a difference in outcome measures between the groups, the duration of treatment was not matched in both the groups, thus there is a possibility that the experimental group did better due to more treatment time.

An RCT done by Narayan Arya et al., recruited 103 mild to moderately affected stroke clients, and randomly assigned them to either the experimental group or the control group (11). The experimental group practiced tasks like lifting a glass, bringing it to the mouth, imitating activities like bathing, washing utensils, etc., The control group received Brunnstrom and Bobath neurodevelopmental techniques for a similar amount of time, i.e., one hour a day, 5 days a week for 4 weeks. The Fugl Meyer Assessment (FMA) for upper extremity, the Action Research Arm Test (ARAT) and WMFT were the outcome measures. The FMA is a quantitative measure to assess upper limb functions specifically post stroke. It tests 5 domains of assessment: motor functions, sensations, balance, joint range of motion and joint pain on an ordinal scale of 3 points from $0-2$, zero being the least functional ability and 2 being normal. This paper focuses on upper limb motor functions, which have a maximum score of 66 (12). ARAT is an observational tool to assess upper limb motor functions after stroke. There are 19 tests, which are further divided into 4 subtests namely: grip, grasp, pinch and gross arm movement. Each test is measured on an ordinal scale of $0-3$, zero means can't perform the test at all and 3 means normal, thus with a maximum score of 57 (13). The experimental group improved on the FMA-UE score 11.98 to 29 , versus 9.88 to 16.42 in the control group showing a between group difference of 9.23 $(\mathrm{P}<0.001)$. Similarly, the ARAT scores, including its sub items (grasp, grip, pinch, and gross 


\section{Development of a Computerized assessment tool for hand-arm function after stroke: test- retest reliability and convergent validity}

movements) significantly changed from 6.56 to 22.84 versus 5.58 to 11.57 having a between group effect of $8.23(\mathrm{p}<0.001)$. The WMFT (time) was found to significantly decline from $97.57 \mathrm{~s}$ to $56.76 \mathrm{~s}$ for the experimental group versus $106.28 \mathrm{~s}$ to $94 \mathrm{~s}$ for control, having the between group difference of $28.7 \mathrm{~s}(\mathrm{p}<0.001)$.

Kwakkel et al., recruited 222 sub-acute stroke clients with at least $10^{\circ}$ of active extension of the wrist, thumb and two fingers, adequate balance, and standing ability to apply CIMT while wearing the mitt (14). 106 clients were randomized to CIMT program and 116 to the usual care and were given their respective treatments for 2 weeks. CIMT group practiced tasks that replicated activities of daily living for example, lifting utensils, pouring water from the jug in a glass, etc., for 6 hours a day. The control group, whereas ranged from no treatment to application of orthotics and typical physical therapy having a different range of motion, stretching, strengthening exercises, etc. The time for which both the groups received treatment did not match, thus there was a huge difference in treatment time and also a difference in quality of the treatment. On WMFT, CIMT group performance time was 34\% more than the control group (95\% confidence interval (CI) 12 to 51). Whereas for the MAL (amount of use) the CIMT group was 0.43 points more than the control (min to max score: $0-5)(95 \%$ CI 0.05 to 0.80$)$; and the MAL Quality of Movement by 0.48 points (min to max score: $0-5)$ (95\% CI 0.13 to 0.84 ). CIMT group also had a greater decrease in self-perceived hand function, difficulty on the SIS hand domain, by $9 \%$ (95\% CI 0.3 to 19). Effect size is not calculated in this study. Although an improvement was seen in CIMT group, there was still a mismatch in the duration and amount of therapy given to each group. This could have been one of the reasons why CIMT group showed more improvement. 


\section{Development of a Computerized assessment tool for hand-arm function after stroke: test- retest reliability and convergent validity}

An RCT done by Azab et al., in 2009 investigated the effectiveness of CIMT on the Barthel Index (BI) scores in mild to moderately affected stroke clients (15). BI is a measure of functional disability in neurologically affected clients. It measures the functional dependency and mobility. 10 daily living tasks such as eating, bathing, grooming, etc. are rated on a scale having three levels, 0, 5 and 10. Zero signifies total dependency; five signifies partial dependency and 10 means full independence. It has a maximum score of 100 (16). Twenty-seven stroke clients were placed in the experimental group, and 17 in the control group. Both the groups were given traditional physical and occupational therapy involving range of motion exercises, stretching, strengthening, etc. The experimental group also received CIMT where the less affected upper limb was placed in a removable cast for 6 hours a day for 4 weeks. Thus, the treatment time and intensity of the treatment were not similar in both groups. The movements practiced were selected from the activities of daily living such as lifting objects, eating and drinking. The BI scores for the control group before and after therapy increased from 68 to 79, whereas for CIMT group they increased from 70 to 97 . Baseline scores were almost similar for both the groups. Post assessment, between group BI scores difference were 18 points. Though a difference between the groups was observed, but was it because of CIMT or due to the higher dosage of therapy given to the experimental group was not justified. Also, BI is not a measure dedicated only to the hand functions; instead it has various components of general body functions and independence. Thus, a measure like the WMFT or MAL would have been a better outcome measure for observing the upper limb functional recovery in specific.

Similar findings were found in several other studies (17), (18), (19) and (20). All these studies compared the effects of CIMT over traditional physical therapy, and reported better results in the former approach in terms of upper limb functions. These findings culminate to the 
Development of a Computerized assessment tool for hand-arm function after stroke: testretest reliability and convergent validity

fact that currently there is enough evidence available to show that task specific, and repetitive movements do improve upper extremity functional performance post stroke. CIMT still has some limitations. Firstly, only mild to moderately affected stroke clients can practice this approach, there is almost nothing for the severely affected clients (18). Secondly, there has to be a one to one guidance during this therapy, as it is not very safe to restrain the good hand of a stroke client and let them be by themselves. Thirdly, only gross movements of the shoulder and elbow could be practiced with this program, there is no consideration given to the fine finger and hand movements. Lastly, after some time this therapy program becomes boring, tedious, monotonous and hard to stick to. These problems make this program impossible to be transferred to home (21), (22).

Though CIMT forms the core basis for neurorehabilitation, i.e., high intensity, high volume and task-oriented practice, adherence is still low because of the above-stated problems. Thus, there is a great need to develop a program, which can overcome these limitations and make physical rehabilitation more efficient, engaging, cost effective, fun and easily transferable to the home. 
Development of a Computerized assessment tool for hand-arm function after stroke: testretest reliability and convergent validity

\section{Technology assisted therapies}

Most stroke clients clearly benefit from intensive inpatient and well-resourced outpatient care (23), (24). Physical rehabilitation post stroke is a slow and tedious process, and most of the clients have to continue rehabilitation for an extended period. There are lots of reasons for low adherence to rehabilitation such as money, transportation, losing interest in the exercises at home after some time, frustration, etc. To try and cope with these problems, a lot of consideration is made for coupling digital media with exercises (25), (26).

Coupling exercises with digital media, more specifically computer games is one way to deal with most of the problems stated above and providing adherence to a high intensity quality therapy, which could be done for a longer period of time, and most importantly could be transferred to the participant's home without making the participant lose their interest (27). Technologies such as the Wii and the Kinect have been explored in the rehabilitation field for quite some time now. 


\section{Development of a Computerized assessment tool for hand-arm function after stroke: test- retest reliability and convergent validity}

\subsubsection{Wii gaming system}

The Wii gaming system is comprised of a wireless remote known as the Wiimote, which has a gyroscope and an accelerometer and detects the user's movements in a 3D plane. An avatar, for example, a tennis racquet is slaved to the movements of the Wiimote, which is held by the participant (28). Various commercial games like bowling, boxing, tennis, etc., can be played with this setup. The movements practiced while playing these games involves gross "swing type movements" i.e. gross movements of the shoulder and elbow, or movements performed just by the hand and the wrist. The Wiimote does not differentiate different upper limb joints and segments and their specific movements. Thus any joint could happen to move it. Further, movement precision and accuracy is minimal with this setup. Also, for grasping the Wiimote participant requires fine control of thumb to press the go button and release it to swing or serve.

All these games involve movement initiation, but where to precisely stop cannot be practiced. On the contrary, while performing the daily living activities, we are required to initiate as well as end the movement at a particular time and position. As a participant needs to hold the Wiimote while playing the games, it is not possible to practice fine manipulation of the hand and fingers. Also, post-stroke there is a tendency for the hand to go into flexion, thus any approach that will help in opening up the hand would be more beneficial rather than enhancing further grasping and closing of the hand. Lastly, the Wii has little value for severely affected participants, as they are required to lift the whole arm against gravity and move to play the games.

Saposnik et al. conducted a Wii based study in 22 mild to moderately affected acute stroke clients. Participants were randomized either to the Wii group or the control group (29). They studied the feasibility as well as the efficacy of the Wii system over recreational therapy. 


\section{Development of a Computerized assessment tool for hand-arm function after stroke: test- retest reliability and convergent validity}

The treatment group played sports games (like tennis, golf, baseball, etc.) with the Wii system; on the other hand, the control group played card games and bingo. Both groups received eight sessions of one-hour therapy over a period of 14 days. Ideally, the movements that should have been practiced while playing Wii games would have been large shoulder and elbow movements, but we are not certain about the same as the accelerometer based Wii remote could be moved by a small flick of the wrist or the forearm, i.e., a trick movement could easily be made. The primary outcome measure was the WMFT in which the treatment group took on an average 7 seconds less than the control group post therapy. On the other hand, there were no significant differences seen in the secondary outcome measures, i.e., the Box and block test, SIS and grip strength. The Box and block test measures gross manual dexterity as the participant is asked to move as many blocks as he/she can from one box to another in one-minute time. A total of 150 blocks are placed in the first box. Greater the number of blocks moved, the better is the performance (30). As there were no adverse events and all the participants could complete the program, a Wii program might be a feasible approach for rehabilitation in acute stroke clients. Although, to achieve better results, an approach that involves movement accuracy, avoids associated movements and avoids further fingers hand flexion should be developed.

Hijmans et al. developed a bilateral hand held Wii gaming therapy (28). They enrolled 14 acute stroke clients; the level of severity was not mentioned in the paper. All the participants were given a control treatment for two weeks in which they played three computer games with the help of a computer mouse. After a washout period of two weeks, the same participants were given bilateral Wii gaming therapy. An accelerometer based Wiimote was attached to a custom made rod, which the participants held with both hands to play games. The movements practiced while playing the games should be with shoulder and elbow, but as said in the above article the 
Development of a Computerized assessment tool for hand-arm function after stroke: testretest reliability and convergent validity

cursor could have been moved with trick movements too. Also, the extent of participation of the less affected hand/arm is unclear. It is possible that the less affected hand/arm did all the movements. Participants were assessed with the FMA, WMFT, and the Disabilities of Shoulder and Hand questionnaire (DASH) 4 times, i.e., before control treatment, after control treatment, before Wii therapy, and after Wii therapy. DASH is a self-report questionnaire that measures upper limb musculoskeletal disability. There are 30 questions, which have to be answered by the client on a scale of $1-5$, one being normal and five being unable to perform at all (31). The FMA was 44.2 at the baseline, 44.0 after control treatment, 45.0 before Wii therapy and 49.2 Post Wii therapy. The other two outcome measures remained almost unchanged (WMFT changed from $32.4 \mathrm{~s}$ after control to $30.5 \mathrm{~s}$ after the intervention, and DASH changed form 54.5 after control to 55.6 after intervention). No significant changes in the WMFT and DASH are likely because only gross arm movements were practiced rather than functional movements. Also, one of the games demanded fine finger hand manipulation. It was one of the few studies that placed importance on the bilateral upper limb movements still, a lot could be improvised and done in the future studies for better results. 


\section{Development of a Computerized assessment tool for hand-arm function after stroke: test- retest reliability and convergent validity}

\subsubsection{Kinect System}

Kinect uses an Infrared (IR) camera that can sense full depth and is used to detect user's full body movements in a 3D space, and thus can interact with the computer screen and games available. The infrared camera is attached to a personal computer using a USB. Unlike the Wii, the Kinect system does not require users to hold any kind of remote or sensor in order to interact with the system and play the games. A calibration is done before playing any game, and for this the participant is asked to stand about two meters away from the Kinect sensor with their arms up for about a few minutes. The IR camera captures the body movements and converts them into the movements of a slaved avatar on the computer screen to play the games. The participant could relate to a full body avatar on the computer screen in which he or she can move their both arms and both legs, i.e., each limb/body segment as one unit. Unfortunately, not every joint can be detected with all their possible movements. For instance, while playing tennis, lifting the racquet can be done by shoulder elevation, elbow flexion or just wrist movement. Further, Kinect is responsive to smooth and steady movements, which are quite unlikely to happen in the clinical population. Further, it can sense large movements of the arms, but fine manipulation with the fingers and hand is still not possible. Also, calibration can be a problem in clients who are unable to move their arm up and hold it for some time. Some of the studies that have used the Kinect system for upper limb stroke rehabilitation are discussed below:

Sin \& Lee studied the additional effect of technology-based therapy using the Kinect system with other conventional therapies such as active ROM exercises and gross dexterity exercises in stroke clients (32). 40 mild to moderately affected participants with in the acute stage of stroke recovery were enrolled in the study and were randomized either in the control group, which only received conventional therapy or in the intervention group, which received 


\section{Development of a Computerized assessment tool for hand-arm function after stroke: test- retest reliability and convergent validity}

conventional therapy plus therapy with the Kinect system. Participants played various games with the Kinect system like bowling, boxing, etc., which focused on the gross movements of the shoulder and elbow respectively. The performance was measured with the FMA and the Box and block test. The FMA for intervention group changed from 26.06 to 47.72 versus 32.29 to 34.49 for the control. Further, the Box and block test scores changed from 11.11 to 20.67 versus 13.59 to 16.29 for the control. Calculation of between group statistics was not mentioned in the paper, which would have been a better way of presenting results. Even with the significant difference observed in the outcome measures, the entire credit cannot be given to the Kinect system therapy as the experimental group overall had more treatment duration. It might be possible to obtain similar outcomes if both groups were given therapy for the same amount of time.

\subsubsection{Marker-based systems}

Two types of marker based movement detection systems have been used in the rehabilitation setups so far. The first system uses passive color markers in which movements are tracked by a vision based tracking system. Different color markers are attached to the body parts of the participant, and a camera is used to detect the movements of these markers. This camera is further connected to a computer. The participant can see a game controller on the computer screen, which moves as and when the color markers attached to body parts move. Thus, there is not one game cursor. Instead, a whole limb can be viewed on the screen. Mostly red, green and blue colors are chosen for data input images into the software, which translates the identity of these colors in dynamic environments. The program starts displaying movements on the screen as soon as the camera captures the images of the color patches. Calibration is done, and all the color markers are identified through a filter to detect only the color patches. Once calibration is done, the participant can start the task. Commercially available games cannot be played with this 


\section{Development of a Computerized assessment tool for hand-arm function after stroke: test- retest reliability and convergent validity}

system. Instead, custom-made tasks are practiced. Also, the sensitivity of the color marker based system is very limited when compared with the systems like Kinect. Further, multiple markers and their movements are translated at the same time; which could be complex for stroke clients to understand when and what joint to move. Also, there are limited options for the movements of small joints of fingers and hand. Lastly, all the movements have to be performed antigravity as there are no such objects that could support the arm during the tasks and eliminate the gravity. Thus, only mildly affected participants can participate in the program.

The second system uses active light emitting diodes (LEDs) markers instead of a camera and color based markers. For instance, in one setup a glove with LEDs attached on its fingertips was used. Two Nintendo Wii remotes were used to track and translate the movements of LEDs on a computer screen in order to perform the task.

Movements are caught in a 3D environment, and a game controller is slaved to move while playing the games. Again, commercially available games cannot be played with these systems. Thus, custom-made tasks are incorporated.

Da Silva el al. in 2011 developed a Rehabilitation Gaming System (RGS), which captured the movements of the upper limb via colored markers attached to the hand and elbow (33). An avatar mimicked the movements of the markers through which the participants were able to interact with the system. Two groups were made, and 17 sub acute moderately affected stroke participants were randomized. The intervention group practiced a task called the Spheroids. In this task, the participants had to interact with the spheres appearing on the screen by grasping, moving and hitting them. The control group played other games with Wiimote or performed similar kinds of manipulation task but without visual feedback. Both the groups were given their respective treatment for three 20 -minute sessions per week for 12 weeks. At the end 


\section{Development of a Computerized assessment tool for hand-arm function after stroke: test- retest reliability and convergent validity}

of 12 weeks, both groups showed an average improvement in the BI and FMA with no significant improvement of any group over the other. This study did not show any treatment approach superior to the other it still shows the feasibility of RGS system in stroke rehabilitation, as the experimental group was able to complete the program with no adverse events. A powered RCT might bring out the efficacy of one system over the other.

Standen et al. published another study using a system, which consisted of a glove with mounted LEDs (34). It was a feasibility study in which the setup was done in the home for 17 sub-acute, mild to moderately affected stroke participants. Participants were asked to wear an LED glove and play three games for three times a day for 20 minutes each for eight weeks. The Wii system recorded the frequency and duration for which the participant played the games. The movements that were practiced while playing the games were forearm pronation-supination, and grasping movements involving finger flexion. The game data was collected for each participant including duration of use; how many times per day the glove was used and a number of days for which the glove was used. Surprisingly out of 17 participants, 4 dropped out, as they did not like the program. It was noted that out of 13 enrolled participants, 11 participants used the glove for less than $25 \%$ of the recommended duration, and 8 participants practiced with the glove for less than $50 \%$ of the recommended number of days. When asked about the barriers while using the glove at the recommended duration 5 participants reported technical issues with the system, and 2 participants reported dependence on family members to help with the equipment. Other reasons given were: health issues, family commitments, and loss of interest after some time. Only 5 participants said they found the games motivating and alleviating boredom. Two of the three games focused on grasping, which post-stroke usually develops, more focus should have been given on extension of hand and fingers. A small sample size with many dropouts is another issue 
Development of a Computerized assessment tool for hand-arm function after stroke: testretest reliability and convergent validity

with this study. Lastly, the setup and equipment were not user-friendly and required major assistance from other family members. Also, a lot of participants encountered technical problems, which lead to unwillingness and irritation in the participants. 


\section{Development of a Computerized assessment tool for hand-arm function after stroke: test- retest reliability and convergent validity}

From the above-discussed studies, it is clear that technology in rehabilitation is still evolving, and a lot of improvisation has to be done in these programs for better results regarding adherence and functional recovery. A lot of limitations are observed in the technology assisted rehabilitation setups, such as most of these setups cost a good amount of money, and are quite complex and cumbersome for the rehabilitation purposes which makes their home transition impossible. Also, the technology used is not that precise and fast to measure or track small finger-hand movements. Further, there is no real object manipulation in these tasks, which eliminate the real handling, touch, and perception of the objects. Movement precision is another significant factor, which is left untouched in these studies. Movement initiation and ending with precision are two different faces of a coin and should not be considered as one. It is easy to perform a random gross movement when compared to a movement that requires accuracy and precision. Any household job requires movement initiation and ending at a specific time and position in space. A little deviation from the actual position can lead to complete disruption of the function. For example, while eating, we have to be very precise that the spoon having food goes into our mouth. A slight deviation from the actual movement path can lead to a complete inability to eat.

Only a few custom made games/tasks and movements could be practiced with these programs as they are not compatible with commercially available, inexpensive games. Thus, there are no such upgrades possible with these programs as they are possible with commercial games. Pastor in 2012 concluded that due to the high complexity of these systems, the requirement of big spaces, and their installing and usage make these approaches impractical for the clinic as well as for home transition (35). 
Development of a Computerized assessment tool for hand-arm function after stroke: testretest reliability and convergent validity

The effectiveness of a therapy program depends on the changes observed in an outcome measure. With the development of a therapy program, it is also important to develop an assessment tool that can measure its effectiveness. Different assessment tools are available to measure upper extremity motor function post-stroke.

A number of performance-based assessments are available such as the WMFT, Box and block test, MAL and DASH. These tests measure hand functions by either the time taken to complete a task or by quantifying the tasks by general observation. Time-based measures have limited value because time alone fails to provide information about the quality, efficiency, and accuracy of a movement. Other tests that utilize general observational skills to quantify movement performance do not provide objective outcomes, as observation can be subjective and differ from person to person. In addition to these limitations, only a few objects have been tested in these assessment tools, the most common being pegs moved or can lifted, or cards turned (36). 


\section{Development of a Computerized assessment tool for hand-arm function after stroke: test- retest reliability and convergent validity}

\subsection{SUMMARY}

Stroke is a leading cause of upper limb disability and rehabilitation post stroke is long lasting and tedious. Various studies have shown the sooner the physical rehabilitation starts post stroke; the better are the outcomes (7). Rehabilitation post stroke is based on the principle of high intensity and high volume of functional exercises (8). CIMT is a paradigm that has been proved to be effective and better than the other traditional therapies in post-stroke rehabilitation (11), (3). Unfortunately, adherence to CIMT is still low due to financial barriers, lack of motivation and interest etc. (4), (37). To improve the adherence of physical exercises, many researchers are using digital media (computer games), such as Microsoft Kinect, Wii, etc. These systems have been tested for their feasibility in clinical setups and proven to be a good starting point (28), (32), (35). Other marker-based systems have tried to develop specific tasks for rehabilitation using digital media (33), (38). Though these systems have taken an initiative to make the rehabilitation world more engaging, there are some limitations associated. For instance, not every joint and movement can be practiced with these systems, only mild to moderately affected stroke clients can benefit from them, the technology of these programs is not user friendly, therefore cannot be transferred to participant's home. The cost of these setups is another issue.

To overcome the above problems, we have developed a set up called the Game based Tele-Rehabilitation (GTR) system, where various objects can be manipulated, and almost any commercially available computer game can be played. The objects chosen are according to the need of the individual client, and so are the games. When practicing with the GTR system, the participant is performing daily functional activities. This system is built on the concept of high 
Development of a Computerized assessment tool for hand-arm function after stroke: testretest reliability and convergent validity

intensity and high volume of quality functional exercises, which can be easily transitioned to the patient's home.

Another good thing about the GTR is that it has an inbuilt Game based Hand-Arm function assessment tool (GHA), which automatically logs in the client's data and various performance measures such as movement time, response time, success rate, etc. are calculated. The GHA provides a full description of how well the participant has performed or improved from time to time. Unlike other assessment tools, which focus on time taken, range of motion or number of objects moved, this tool focus on movement quality in terms of accuracy and precision. 
Development of a Computerized assessment tool for hand-arm function after stroke: testretest reliability and convergent validity

\subsection{PURPOSE OF STUDY}

The overall goal of this study was to develop a home based Tele-rehabilitation platform and test the psychometric properties of the embedded assessment tool for hand and arm functions post stroke.

To fill in the gaps present in the current upper extremity assessment tools for stroke participants it is important to develop a tool, which quantifies the upper extremity functions by movement quality, accuracy, and variance. Also, a tool, which is not restricted to the testing of a few objects, like pegs and coins, but has a broad range of object-manipulation. 


\section{Development of a Computerized assessment tool for hand-arm function after stroke: test- retest reliability and convergent validity}

\subsection{THE GTR/GHA SYSTEM}

The GTR system consists of an innovative game-based exercise system through the novel use of commercial computer input devices, which takes advantage of the wealth of inexpensive and easily accessible commercial computer games. An inexpensive, wireless Air-mouse (The Scoop $^{\text {TM }}$ Pointer Remote Model: RXR1000-0302E, Hillcrest Labs) is used to play the games. The air mouse uses inertial sensors to derive angular displacement and allows the physical motion to be translated and interpreted. Many objects or utensils can be modified to function exactly as a computer mouse simply by attaching the small Air-mouse using Velcro. The approach is intended to provide engaging, high-quality therapy with monitoring of function in the home via Tele-rehabilitation and supported by clinicians. The GTR system focuses on a goaloriented approach according to every individual and their impairments, which relates to their participation in real-life situations. Depending on physical and ergonomic properties of everyday objects various tasks can be practiced with the GTR system. Knowledge of the therapeutic values of these objects and tasks can assist the therapist to prescribe graded exercises and target specific functional goals. This innovative approach provides a highly flexible and personalized treatment tool. Not only the objects and tasks, but also the choice of computer games have particular therapeutic value. Different computer games require varying levels of movement amplitude, speed, accuracy and repetition. Approximately one hundred of easily accessible, inexpensive commercial computer games have been tested with different objects. Thus, a broad range of objects as well as computer games is available for practicing different functional activities. These activities require manipulation of objects with a wide range of physical properties (size, shape, weight, inertia, location of mass center), and often require a high degree of precision, where small deviations in timing or end point positioning/orientation of the object leads to complete 


\section{Development of a Computerized assessment tool for hand-arm function after stroke: test- retest reliability and convergent validity}

disruption of performance. By focusing clients' attention with games that require coordinating the movements and controlling diverse objects as game controllers, their skills will then improve gradually. The utmost important part is that due its simple hardware and software, the GTR could be easily transferred to the participant's home and provided quality therapy with telesupport.

At the core of the GTR are interactive, computer-based rehabilitation games with automated monitoring tools for enhanced therapeutic exercises that target fine and gross motor skills of the hand and arm. This not only provides an improved therapy program but also records exercise duration and performance outcomes through the inbuilt assessment tool, the GHA. The GHA provides a standardized method to objectively quantify task performances during manipulation of a broad range of everyday objects having different (a) physical and surface properties, (b) precision level and (c) function (anatomical requirements, i.e., 2-finger, 3-finger, whole hand or bilateral tasks). The game assessment software automatically logs the client's performance and computes their movement quality, efficiency, and accuracy for each manipulation task. This addresses the challenge of quantitative and objective outcome measurement and provides the means to obtain electronic records for Tele-rehabilitation.

The idea of a tool in which treatment and assessment are integrated is very attractive as it is efficient and time saving, documents volume of practice; provides a measure of intensity, duration, and compliance, allows tracking of change within each treatment session and over time thus allowing examination of "dose-response" relationship. It also provides timely feedback and support for client and clinicians. Electronic records and reports can be generated to inform therapists when and how a participant is performing, whether they are experiencing any difficulty, and how they are progressing in their individualized home exercise program. This 
Development of a Computerized assessment tool for hand-arm function after stroke: testretest reliability and convergent validity

opens up an exciting possibility for affordable, and scientifically motivated improvements in stroke rehabilitation. 
Development of a Computerized assessment tool for hand-arm function after stroke: testretest reliability and convergent validity

\subsection{RESEARCH OBJECTIVES}

This study had three objectives:

1. The first objective was to evaluate the test-retest reliability of the GHA assessment tool embedded in the GTR system by computing the ICC values from test one and two.

2. The second objective was to evaluate the convergent validity of the GHA assessment tool embedded in the GTR system by comparing the outcomes of GHA with WMFT and grip strength, specifically to assess the fine and gross motor skills in individuals who have suffered a stroke.

3. Third and last objective of this study was to determine the known group validity of GHA performance measures for each object manipulation task by comparing the stroke group performances with that of the healthy group.

\subsection{HYPOTHESES}

This study had three hypotheses:

1. The first and the working hypothesis was that the GHA performance measures would show high test-retest reliability in terms of high ICC values.

2. The second hypothesis was that the GHA performance measures would show a low to moderate correlation with the WMFT and grip strength.

3. Third and the last hypothesis was that the healthy group would perform significantly better than the stroke group in terms of the GHA performance measures. 
Development of a Computerized assessment tool for hand-arm function after stroke: testretest reliability and convergent validity

\subsection{METHODS}

Ethics Approval

The University of Manitoba human research ethics board and Riverview health center ethics committee issued the approval for the study.

\subsubsection{Sample Size}

A sample size of 30 acute/sub-acute stroke inpatients/outpatients was recruited for the test-retest reliability and convergent validity of GHA. Also, 20 healthy subjects between of 2030 years of age were recruited for the between-group comparison. The healthy individuals were all graduate students at the University of Manitoba and had no history of neurological impairments, vision problems or hearing problems.

\subsubsection{Inclusion Criteria}

Stroke clients were recruited if they had the following inclusion criteria:

(a) Age 40-70 years.

(b) Time since stroke less than 2-years.

(c) Ability to actively extend at least 10 degrees at the metacarpophalangeal and interphalangeal joints and 10 degrees at the wrist joint. At least 30 degrees of active flexion-extension at the elbow and some active movements present at the shoulder joint.

(d) English speaking, no clinical evidence of cognitive impairment (screened by a physician or therapist), and able to provide informed consent. 
Development of a Computerized assessment tool for hand-arm function after stroke: testretest reliability and convergent validity

\subsubsection{Exclusion criteria}

Stroke clients having excessive spasticity, pain, or contractures were not included in the study. Convulsive seizures preventing adequate attention to the tasks, problems with vision, aphasia and apraxia were also considered as exclusion criteria. 


\section{Development of a Computerized assessment tool for hand-arm function after stroke: test- retest reliability and convergent validity}

\subsubsection{Testing Protocol}

Test-Retest reliability and Convergent validity of the GHA assessment tool-

Szturm et al. have demonstrated reliability and validity of the GHA in 20 young, healthy individuals (baseline control data) (39), and as well as in patients with hand arthritis. The test protocol indicated moderate to high test-retest reliability of performance measures for three manipulation tasks. ICCs were obtained ranging from 0.5 to $0.84(\mathrm{p}>0.05)$ for the people with hand arthritis (36). The present study extended the test-retest reliability in the individuals with stroke.

30 clients with upper limb hemiparesis and impairment caused by stroke were recruited through their attending Physician, Physiotherapist or Occupational Therapist at the Riverview Health centre or Health Sciences Centre in Winnipeg, Canada. Ten different GHA tasks were performed for each participant.

The following hand function assessments were conducted and used to examine the convergent validity of GHA performance measures:

1.) Quantitative measures of upper extremity functional ability through 15 timed tasks were measured by WMFT.

2.) A grip strength test, using a dynamometer was performed to test the general strength level of hand and wrist muscles.

The first session included administration of the WMFT followed by 10 GHA tasks. The WMFT has 15 time-based tasks (44). In the first session 8 of the 15 tasks were performed. The participants were explained each task before administration. 120 seconds were given to complete each task, but at the same time the participants were asked to complete the task as soon as they can. Tasks unable to perform were given a score of 120 seconds. Also, every task was graded on 
Development of a Computerized assessment tool for hand-arm function after stroke: testretest reliability and convergent validity

an ordinal scale of 0 to 5 , where 0 indicated did not perform at all and 5 indicated carried out with normal movement. 10 GHA tasks were administered after WMFT. The participants were comfortably seated and an adjustable table where objects were placed and manipulated was put in front. A computer monitor was placed 1.5-meters in front of the participant at the eye level. All the participants were provided with a demonstration of testing. After one practice trial of 15 seconds, each task was recorded. Three to four days apart, the second test was performed and remaining 7 tasks of WMFT were completed, followed by grip strength testing of the more affected hand. Grip strength was tested using an isometric hand dynamometer (G100, Biometrics Ltd., UK) in the testing position recommended by the American Society of Hand Therapists. The average score of the two consecutive trials was used for the analysis. All the GHA tasks were again performed in the similar manner as done in the first session. Also, for the between group comparison of GHA performance measures, 20 healthy subjects performed the same GHA tasks in one session and their performances were compared with the performances of 20 randomly chosen stroke participants. 


\section{Development of a Computerized assessment tool for hand-arm function after stroke: test- retest reliability and convergent validity}

\subsubsection{Materials}

The wireless Air mouse was used to play the assessment games. As the Air mouse can be easily attached with Velcro to many objects, GHA provides a highly flexible assessment tool applied to fine or gross upper extremity motor skills. GHA further has following two assessment modules that has been developed and were tested in this study:

\subsubsection{Cyclic tracking module}

This involved cyclic tracking of a visual target that moved horizontally left to right or vertically up and down on a computer display for several cycles. Two cursors of different shape appear on the monitor. One is the "target" cursor (motion is computer controlled). The target cursor moves at a predetermined frequency and amplitude (user defined e.g. $0.5 \mathrm{~Hz}$, amplitude of $70 \%$ of monitor width). The second "game" cursor is "slaved" to the motion of the air mouse. The air-mouse was attached to various test objects and in this manner motion of the game cursor represents the movement of the test object. The client was asked to move the game cursor in synchrony with the moving target cursor for 60 seconds or approximately 25 repeated back and forth movements of the test object. The computer application records the position coordinates of the target and game cursors at a sampling rate of $80 \mathrm{~Hz}$. This logged game data is used to quantify performance of each object-manipulation task. For more information refer to figure 1.

\subsubsection{2. Motor skill Game Module}

The goal of this computer task was to move a paddle (the game sprite) to interact with moving objects that appeared at random locations on the computer screen. The speed of the target objects and the size of the game paddle (precision level) were configurable. The time 


\section{Development of a Computerized assessment tool for hand-arm function after stroke: test- retest reliability and convergent validity}

period between successive target appearances is also configurable. Typically 2-3 seconds is used. Thus, for one test session of 2 minutes, 40-60 player movements are made; one-half in each direction (left-right or up-down with one-half of medium amplitude and other of large amplitude). Again, the test objects are equipped with the air-mouse and controls the position/motion of the game cursor. The computer application records the game data at a sampling rate of $80 \mathrm{~Hz}$. This logged game data is used to quantify the following performance measures, movement response time, movement duration, movement accuracy, movement efficiency, and movement variation over the 40-60 movement events. For more information, refer to figure 2 .

\subsubsection{Object selection}

Many objects of daily life can be instrumented with the air- mouse and hence a myriad of functional tasks can be examined. The following object-manipulation tasks were chosen to test GHA:

1. Flexor spasticity in fingers and hand is a major problem post stroke and exercises that promote finger extension are important. In the first task we attached the air-mouse on a cylindrical object (diameter of $10 \mathrm{~cm}$ ). Game play required the client to roll the cylinder back and forth using their fingertip. This task promoted finger extension. Apart from the finger extension, wrist, elbow and shoulder flexion-extension were also practiced in this task.

2. To increase the difficulty level a soccer ball was used for rolling. This time the participant had to have more control as the soccer ball allows 2 degrees of freedom, i.e. it could be moved in more than one plane whereas; the cylinder only had one degree of freedom and could be only moved in one plane. The participant rolled the soccer ball right and left by placing the hand on 


\section{Development of a Computerized assessment tool for hand-arm function after stroke: test- retest reliability and convergent validity}

the top of it. The movements performed were internal and external rotations of the shoulder, some flexion-extension of the elbow, and extension of the wrist and fingers.

3. A joystick was crafted for practicing the large movements of shoulder and elbow. It was kept on the affected side of the participant at an adequate height so that the participant's hand was at knee level while sitting on a chair. Further, the Air mouse was attached at the bottom of the joystick. The participant manipulated the joystick by placing the hand on the top and moving it forward and backward. This task also focused on the hand and wrist extension.

4. In the fourth task we attached the motion mouse on a coffee mug, which was held with a power grip and rotated side to side to control the game cursor position/motion. Elbow pronation and supination were practiced in this task.

5. Fine control movements of the thumb and fingers are extremely important for many activities of daily living. These functions range from (a) two-finger functions, e.g. turning a key or coin, (b) three-finger function, e.g. turning a doorknob. For this purpose a crafted set up replicating a doorknob was used. Participant held the doorknob by a 2-finger or 3-finger pinch grip and rotated it in both directions to control the position/motion of the game cursor.

6. A similar setup was made for the whole hand function where an object was crafted which required whole-hand grip. The participant had to hold it and rotate right and left just like opening and closing a jar lid.

7. Bi-manual task: many activities of daily living require manipulation of objects with both hands, e.g. driving, carrying large size objects, mopping, etc. Our last object was a bilateral one in which the participant held and manipulated a beach ball. The task required the client to use both hands and rotate the beach ball to control the position/motion of the game cursor. 
Development of a Computerized assessment tool for hand-arm function after stroke: testretest reliability and convergent validity

These objects represented a wide range of physical properties requiring different manipulation skills, such as pinch grip, power grip, whole hand grasp and bilateral movements respectively. The difficulty level within each object and task could be increased or decreased by altering: 1) Physical Load (for example Torque, Weight, Size, degree of freedom and Gravity), 2) Anatomical Load, i.e., the anatomical movements required and lastly 3) Physiological Load (example, Precision and Accuracy). During both testing sessions, the motion sensor was secured consistently at a point marked in a certain portion of all the above objects.

\subsubsection{Data Analysis}

Cyclic tracking task performance measures

Figure 3C presents synchronous plots of the target motion (circle) and object manipulation (square) performed by a client for a typical GHA tracking task. The quality of movement is quantified by computing the Total residual error (TRE), which is the sum of the difference in position between the computer reference target and the participant position as shown in figure 1. TRE is measured in pixels. Amplitude consistency (AC) is the variation in movement amplitude for each half cycle, i.e. in 20 cycles there would be 20 left to right movement and 20 right to left movement or 20 top to bottom or 20 bottom to top. The amplitude consistency is measured in percentage and is determined as the standard deviations normalized by mean for all the cycles over $60 \mathrm{~s}$. A detailed description of analysis has been previously published (53), (36). 


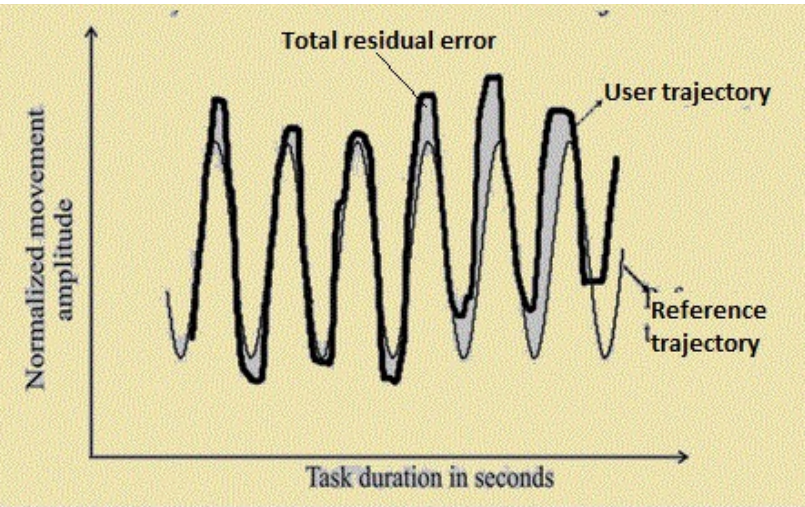

Figure 1-The raw data of cyclic tracking task. The difference between the reference trajectory and user trajectory is the total residual error, measured in pixels.

\section{Motor Skill tasks Performance Measures}

This module records the time of each target object appearance and disappearance and logs the position coordinates of target objects and game paddle at a rate of $80 \mathrm{~Hz}$. Figure $2 \mathrm{~A}$ presents the manipulation of a coffee mug while playing GHA motor skill task. Figure 2B presents the segmented game events. Figure $2 \mathrm{C}$ presents the sorted overlay trajectories of participant movements for all rightward and leftward game events recorded in one game session, and figure $2 \mathrm{D}$ presents the performance measures of this module. The following performance outcomes averaged for all rightward game movements were determined; (a) Movement onset time (MOT): the time from target appearance to start the game sprite (paddle) movement, measured in seconds, (b) Success rate (SR): determined as the percentage of target objects that were caught, (c) Movement variation (MV): it is the standard deviation calculated from the mean of all movement trajectories and calculated in percentage. For a detailed description of motor skill game module refer (54), (55), (56). MATLAB (The Math Works, Natick, MA, version 2010a) was used to compute the outcome measures for the both tracking and game tasks. 
Development of a Computerized assessment tool for hand-arm function after stroke: testretest reliability and convergent validity

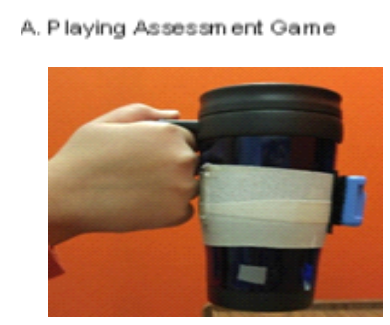

c. Sorted game events

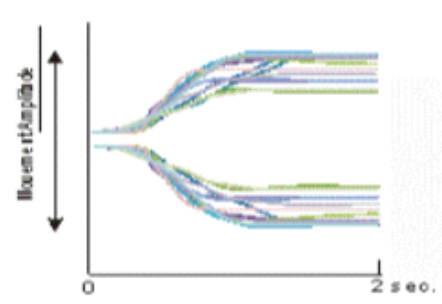

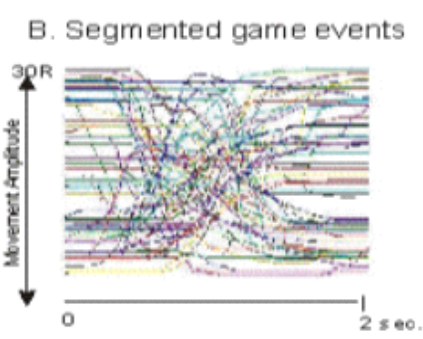

D. Analysis methods

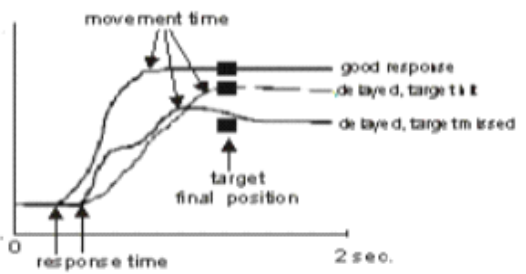

Figure 2-The raw data of motor skill game task. Panel A presents a participant manipulating mug and playing the game, Panel $B$ presents the segmented game events of all left and right directions, Panel $C$ presents the sorted left, and right game events and Panel D presents the analysis method, with time (in Sec) on the $X$ axis and movement amplitude on the $Y$ axis.

\subsubsection{Statistical Analysis}

ICC values were calculated to determine the relative test-retest reliability (57), (58). ICC values were interpreted as very high (ICC $>0.9$ ), high (ICC $>0.7)$, moderate (ICC between 0.50.7) and low (ICC < 0.5) (59). Absolute reliability was calculated using Standard error of measurement $(\mathrm{SEM})$, using the formula $S E M=S D \times(\sqrt{1-I C C)}$ where SD is the average standard deviation of the two session scores (60), (61). Paired student ' $t$ ' test for mean differences between the two sessions scores was also done. The Minimal detectable change (MDC) was calculated using the formula $M D C=1.65 \times S E M \times \sqrt{2}$, where 1.65 is 2-tailed tabled Z-value for $90 \%$ confidence interval and $\sqrt{2}$ is the variance of 2 measurements (62), (63). MDC can be interpreted as the magnitude of change in the performance measures below which there is $90 \%$ chance that the change is due to an error, and no real change has occurred. Thus, any change greater than MDC signifies a real or significant change. 
Development of a Computerized assessment tool for hand-arm function after stroke: testretest reliability and convergent validity

Convergent validity was analyzed using Pearson correlation co-efficient between GHA performance measures and WMFT (time) and grip strength. Spearman rank correlation coefficient (rho) to determine the strength of the relationship between GHA performance measures and WMFT (movement quality). The strength of the correlation was interpreted as high (r/rho > $0.7)$, moderate $(0.4$ to 0.7$)$ and low $(<0.4)$ (10), (64). A within stroke group correlation of GHA performance measures was done by computing Pearson correlation coefficient. Known group validity was evaluated by performing a between group analysis in stroke and healthy groups by using paired Student t-test. Data was analyzed with IBM SPSS Statistics for Windows, Version 19.0. Armonk, NY: IBM Corp. Statistical significance was $\mathrm{p}<0.05$ (two-tailed). 
Development of a Computerized assessment tool for hand-arm function after stroke: test-retest reliability and convergent validity

\section{CHAPTER-2: MANUSCRIPT}

\section{Development of a computerized assessment tool for hand-arm function after stroke: test-retest reliability and convergent validity}

\section{AUTHORS}

Zoya Imran PT, M.Sc. graduate student, College of Rehabilitation Sciences, University of Manitoba.imranz@myumanitoba.ca, Dr. Tony Szturm, PT, Ph.D., Associate Professor, College of Rehabilitation Sciences, University of Manitoba

Dr. Barbara Shay, Ph.D., Head of the Department, College of Rehabilitation Sciences, University of Manitoba

Dr. Sepideh Pooyania, MD. FRCPC, Assistant Professor, Department of Internal Medicine, University of Manitoba, Attending Physician Stroke Rehabilitation Unit, Riverview Health Centre

\subsection{ABSTRACT}

Background: A number of performance-based assessment tools are available to assess functional ability post-stroke, such as Wolf motor function test (WMFT) and box and block test. These tests measure the hand function with either the time taken to complete a task or number of objects moved. These measures have limited value because time alone fails to provide information about movement quality, movement accuracy or movement variation. Also, only the manipulation of a few objects has been tested so far, the most common being moving pegs, lifting cans and turning cards.

Objective: A new computer game-based hand-arm function evaluation (GHA) tool has been established to quantify fine and gross object manipulation skills, movement quality, accuracy, response time, and movement variation. The tool can be used with numerous objects with various geometric/surface properties and functional and anatomical 
Development of a Computerized assessment tool for hand-arm function after stroke: test-retest reliability and convergent validity

demands. The objectives of this study were to determine the test-retest reliability and convergent validity of the GHA assessment tool.

Methods: Thirty stroke clients, the mean age of 68.41 years who suffered a single stroke of thrombo-embolic or haemorrhagic origin, within the previous six to twenty-four months were recruited. Each participant was assessed twice (test and retest) with the GHA assessment tool and once with WMFT. Ten object-manipulation tasks with different functional, anatomical and physical demands were tested. Intraclass correlation coefficient (ICC) values to establish a test-retest reliability of the GHA assessment tool were calculated. Also, the convergent validity of the GHA assessment tool was determined by comparing its outcomes with that of WMFT and grip strength.

Results: ICC values reflected the complexity of the object manipulation task of the GHA assessment tool. The results showed that the more complicated the task, the lower the ICCs and the higher the standard error of measurement (SEM) and minimal detectable change (MDC). Most of the performance measures of the GHA assessment tool had high to moderate ICC values ranging from 0.87 to 0.51 . Also, high to moderate ICC performance measures had lower SEM and MDC values. The Total residual error (TRE) of the mug, cylinder and soccer ball manipulation task had ICC of $0.87,0.81$ and 0.72 and SEM of $8.23 \%, 10.06 \%, 12.64 \%$ and MDC of $19.16 \%, 23.43 \% 29.44 \%$. Scant low correlations were established between GHA performance measures and WMFT and grip strength $(\mathrm{r}<0.7)$. A between group (stroke and healthy groups) analyses showed that the 
Development of a Computerized assessment tool for hand-arm function after stroke: test-retest reliability and convergent validity

healthy group performed significantly better than the stroke group in all GHA performance measures.

Conclusion: This study illustrates the utility of GHA for assessment for fine and gross motor skills. High to moderate ICC values and relatively low systematic errors in the performance measures indicate that this tool can repeatedly measure reliable data from stroke clients. There was a limited correlation between GHA performance measure and the WMFT. This indicates that the two assessment tools represent distinct features or qualities of hand-arm functions. The GHA assessment tool measures the actual movement accuracy, variation and response time of a broad range of fine and gross motor skill task with different functional demands, whereas the WMFT records time to complete a task.

Keywords: hand-arm functional assessment, computerized assessment, test-retest reliability, convergent validity, and tele-rehabilitation 


\section{Development of a Computerized assessment tool for hand-arm function after stroke: test-retest reliability and convergent validity}

\subsection{INTRODUCTION}

Stroke is a leading cause of upper limb dysfunctions (1). Studies have shown that more than $60 \%$ of stroke survivors report persistent hemiparesis resulting in impaired fine and gross motor skills of the upper extremity (40), (5), (6). Manual dexterity involving handling and manipulating objects with the fingers and hand is important for nearly all daily life activities such as dressing, grooming, eating, etc. These activities require object-manipulation of a wide range of physical properties (size, shape, weight, inertia, location of mass center), and often require a high degree of precision where small deviations in timing or end point positioning/orientation of the object leads to complete disruption of performance (2), (7), (8).

Post-stroke rehabilitation is tedious, and full recovery is unlikely (24). It is very import for clinicians and researchers to evaluate the effectiveness of therapies/interventions to track changes before and after, and most importantly to evaluate if one can incorporate a tele-rehabilitation format i.e. high-quality therapy program transferred to the client's home with supervision (41), (42).

An accurate, reliable and valid assessment tool is important to quantify clinical/functional manifestations of mild to severe impairments post stroke. Assessment tools should be able to identify clients' functional status, which could be further used to determine adequate treatment and quantify the changes in upper limb motor functions before and after intervention (43).

A number of assessment tools are available to evaluate upper limb functions post stroke. Some are time-based tools such as the Wolf Motor Function Test (WMFT), which measures upper limb functional ability by recording the time taken to complete 15 tasks 


\section{Development of a Computerized assessment tool for hand-arm function after stroke: test-retest reliability and convergent validity}

and grading the tasks for movement quality on the basis of general observation (44), (9). The box and block test records the number of blocks moved in a one-minute time period from one box to another $(30,45),(46)$. These time-based measures have limited value because time alone does not provide information about the movement quality, efficiency, movement accuracy or types of movement errors. Besides, only a few objectmanipulation tasks have been tested, the most common being moving pegs, lifting cans and turning cards. Some are observation-based assessment tools such as the FMA (12), (47) and ARAT (13), (48). Other widely used tools are self-perception questionnaires or interview-based tools. For instance, the MAL is a semi-structured interview that asks clients to rate the quality and amount of movement for 30 activities of daily living. The tasks evaluated include object manipulation (e.g. pen, fork) and daily activities such as transfers (49), (50). The DASH is an example of a self-report questionnaire. This questionnaire contains 30 items on disability and symptoms related to upper limb activities such as opening a jar, carrying objects, etc. (31), (51), (52). Observational tools and self-perception questionnaires provide a limited picture of functional impairment but may provide an adequate picture of the limitations in life-role activities/participations.

To fill in the gaps present in current upper limb functional assessment tools for stroke, the GHA assessment tool was developed by Szturm et al. (36). Two custom-made computer tasks were developed where a wide range of objects can be manipulated by attaching a motion mouse to them. This attachment makes that object the mouse or game controller. In these two tasks, the participant either tries to track a rhythmic moving target or moves a paddle to catch randomly appearing small targets on the computer screen. The 


\section{Development of a Computerized assessment tool for hand-arm function after stroke: test-retest reliability and convergent validity}

participants perform these tasks by manipulating a wide range of objects, which have different geometrical and surface properties, functional and anatomical demands. These tasks objectively measure movement components like movement quality, accuracy, response-time and success rate. The reliability and validity of the GHA assessment tool has been demonstrated in individuals with hand arthritis (36) and as well as in normal healthy individuals (39).

The first objective of this study was to evaluate the test-retest reliability of GHA performance measures in stroke participants. We hypothesized that GHA performance measures would exhibit high test-retest reliability (ICCs $>0.7)$. The second objective of this study was to determine the convergent validity of the GHA assessment tool. For that, we compared it with the WMFT because WMFT also involves manipulations of objects with different size, shape and functional demands, just like GHA. Still, performance measures of the WMFT and GHA tool focus on different characteristics of a movement; therefore we hypothesized a moderate correlation (Pearson $r$ value between 0.4 and 0.7 ) between them.

The last objective of this study was to determine the known group validity of the GHA performance measures for each object manipulation task by comparing the stroke group performances with that of the healthy group. We hypothesized that the healthy group would perform significantly better than the stroke group for all GHA performance measures.

\subsection{METHODS}

A repeated measure design was used. Thirty participants (20 males and 10 females), age between 40 and 70 years who suffered a single stroke of thrombo-embolic 
Development of a Computerized assessment tool for hand-arm function after stroke: test-retest reliability and convergent validity

or hemorrhagic origin within the previous twenty-four months were recruited.

Participants included had at least 10-degrees range of motion at MCP, IP and wrist joints, 30-degrees of elbow flexion-extension, and some active movements present at the shoulder joint. Also, they were English speaking and had no clinical evidence of cognitive involvement.

Participants with excessive spasticity, contractures, and problems with vision were excluded from the study. The human research ethics board of the University of Manitoba and the Riverview Health Center ethics committee granted ethics approval for the study. Informed consent before the evaluation was taken from each participant.

\subsection{MATERIALS}

Two computerized custom applications were developed which standardized the task used to guide a variety of object-manipulations. This included a small motion sense mouse (The Scoop ${ }^{\text {TM }}$ Pointer Remote Model: RXR1000-0302E, Hillcrest Labs). Various objects of daily life can be instrumented with the motion mouse, and hence different types of functional tasks can be examined. For this study, seven objects were chosen with different physical properties (size, shape, weight, texture, etc.), functional demands (movement precision and accuracy) and anatomical demands (manipulation using 2-finger grip, whole hand grip, bi-manual and involvement of wrist, elbow, and shoulder). The GHA assessment tool included two modules. Table 1 presents the different objects that were manipulated during GHA tasks. It also presents specific joint involvement and their respective movements. Lastly, it presents which mode of the GHA tool (Cyclic tracking or motor skill game) was performed. The objects ranged from large to small ones. Large objects included a soccer ball, cylinder, and joystick, which required 
Development of a Computerized assessment tool for hand-arm function after stroke: test-retest reliability and convergent validity

movements at the shoulder, elbow and wrist. Medium and small sized objects included a mug; jar lid and doorknob, which required movements at the forearm, wrist, and hand. Lastly, there was a bilateral task in which a beach ball was manipulated by the hand, wrist and elbow. 
Development of a Computerized assessment tool for hand-arm function after stroke: test-retest reliability and convergent validity

Table 1- Objects manipulated and their respective movements.

\begin{tabular}{|c|c|c|c|}
\hline Object & Task & Movements involved & GHA mode \\
\hline Cylinder & $\begin{array}{l}\text { Participant placed his/her } \\
\text { hand on the top of a } \\
\text { cylindrical jar and } \\
\text { manipulated it by rolling } \\
\text { forward and backward. }\end{array}$ & $\begin{array}{l}\text { Shoulder and elbow } \\
\text { flexion-extension. Wrist } \\
\text { and hand kept in } \\
\text { extension. }\end{array}$ & $\begin{array}{l}\text { Cyclic tracking } \\
\text { Motor skill } \\
\text { Game }\end{array}$ \\
\hline Soccer ball & $\begin{array}{l}\text { Hand on the top of the soccer } \\
\text { ball and manipulated by } \\
\text { rolling left and right. }\end{array}$ & $\begin{array}{l}\text { Internal and external } \\
\text { shoulder rotations with } \\
\text { some elbow flexion and } \\
\text { extension. Wrist and } \\
\text { hand help in the } \\
\text { extension. }\end{array}$ & Cyclic tracking \\
\hline Joystick & $\begin{array}{l}\text { The hand kept on the circular } \\
\text { top surface of the joystick } \\
\text { and manipulated by tilting } \\
\text { forward and backward. }\end{array}$ & $\begin{array}{l}\text { Large shoulder and } \\
\text { elbow flexion-extension. } \\
\text { Wrist and hand kept in } \\
\text { extension. }\end{array}$ & $\begin{array}{l}\text { Motor skill } \\
\text { Game }\end{array}$ \\
\hline Mug & $\begin{array}{l}\text { A coffee mug held with its } \\
\text { handle through a power grip } \\
\text { of hand and manipulated by } \\
\text { rotating it left and right. } \\
\text { Elbow till mid forearm } \\
\text { supported on a Styrofoam. }\end{array}$ & $\begin{array}{l}\text { Forearm pronation- } \\
\text { supination. }\end{array}$ & $\begin{array}{l}\text { Cyclic tracking } \\
\text { Motor skill } \\
\text { Game }\end{array}$ \\
\hline Jar lid & $\begin{array}{l}\text { Held with all the tips of } \\
\text { finger and thumb and } \\
\text { manipulated by rotating it } \\
\text { right and left. Elbow till mid } \\
\text { forearm supported on a } \\
\text { Styrofoam. }\end{array}$ & $\begin{array}{l}\text { Flexion at PIP and DIP. } \\
\text { Extension, adduction, } \\
\text { and abduction at MCP } \\
\text { joints. Wrist in } \\
\text { extension and some } \\
\text { forearm pronation- } \\
\text { supination. }\end{array}$ & $\begin{array}{l}\text { Motor skill } \\
\text { Game }\end{array}$ \\
\hline Doorknob & $\begin{array}{l}\text { Held with pinch grip of } \\
\text { thumb and index finger, } \\
\text { manipulated by rotating right } \\
\text { and left. Elbow till mid } \\
\text { forearm supported on a } \\
\text { Styrofoam. }\end{array}$ & $\begin{array}{l}\text { MCP, PIP and DIP of } \\
\text { thumb and index finger } \\
\text { in slight flexion with } \\
\text { other MCP, PIP and DIP } \\
\text { in full flexion. Fine } \\
\text { adduction and abduction } \\
\text { of thumb and index } \\
\text { finger with some } \\
\text { forearm pronation- } \\
\text { supination. }\end{array}$ & $\begin{array}{l}\text { Cyclic tracking } \\
\text { Motor skill } \\
\text { Game }\end{array}$ \\
\hline Beach ball & $\begin{array}{l}\text { Held with bilateral hands and } \\
\text { rotated up and down. }\end{array}$ & $\begin{array}{l}\text { Wrist ulnar-radial } \\
\text { deviation with elbow } \\
\text { fixed in } 90 \text {-degree. }\end{array}$ & Cyclic tracking \\
\hline
\end{tabular}




\subsubsection{Cyclic tracking module}

This test involved cyclic tracking of a visual target that moved horizontally left and right or vertically up and down on a computer display for several cycles. Two cursors of different shapes appear on the monitor. As shown in figure 3-B, there is one "target" cursor (motion is computer controlled), which moves at a predetermined frequency and amplitude e.g. $0.5 \mathrm{~Hz}$, amplitude of $70 \%$ of monitor width. The second is the "game" cursor, which is "slaved" to the motion of the air mouse, which is attached to various test objects and in this manner motion of the game cursor represents the motion of the test object, as shown in figure 3-A. The client is asked to move the game cursor in synchrony with the moving target cursor for 60 seconds or approximately 25 repeated back and forth movements of the test object. Figure 3-C presents the plots of the target and user trajectories while manipulating a mug using pronation and supination.

The computer application records the position coordinates of the target and game cursors at a sampling rate of $80 \mathrm{~Hz}$. This mode has two outcome measures, TRE and AC. 
Development of a Computerized assessment tool for hand-arm function after stroke: test-retest reliability and convergent validity

A. Participant manipulating an object attached with a mouse.

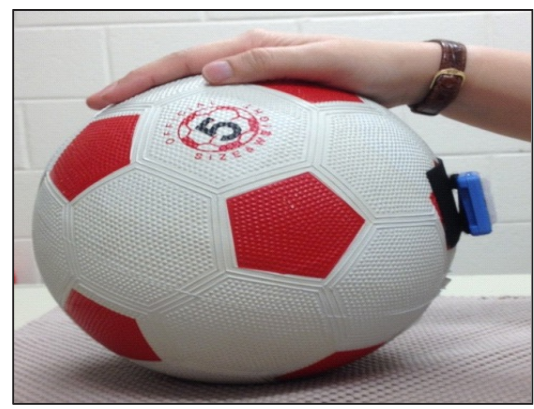

C. Trajectories of computer target and object movement

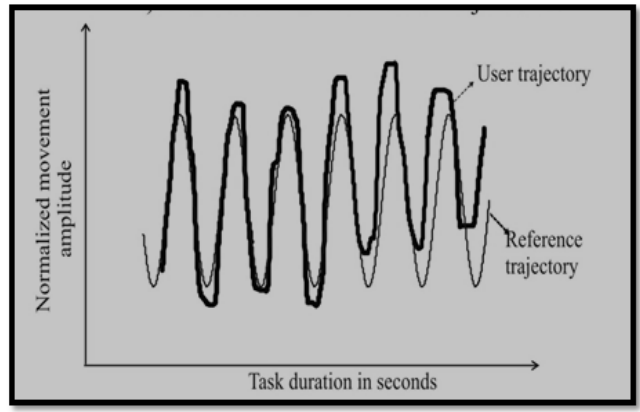

B. Components of tracking task

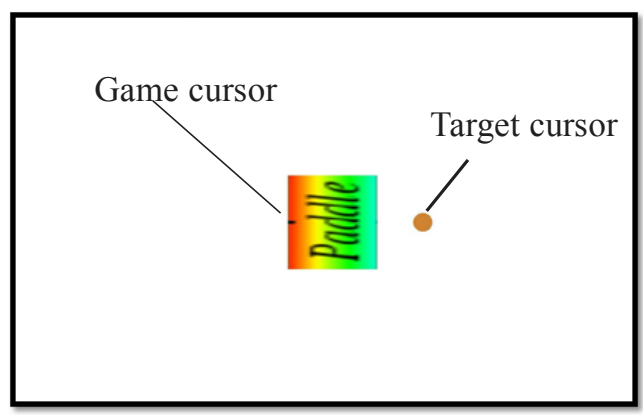

Panel A presents a participant manipulating soccer ball attached with a motion mouse. Panel B presents the components of a tracking task where the paddle is controlled by object manipulation and circle by computer. Panel C presents the synchronous plots of reference cursor motion and user object manipulation for a typical tracking task.

Figure 3 Cyclic tracking task, and its components. 


\section{Development of a Computerized assessment tool for hand-arm function after stroke: test-retest reliability and convergent validity}

\subsubsection{Motor skill Game}

The goal of this computer task was to use the test object to move the game paddle (game sprite) to interact with moving objects that appear at random locations on the computer screen as shown in figure 4-A and 4-B. The speed of target object was slow; it took 3 seconds for the target to move from one edge of the monitor to the other edge. The game paddle size was medium (on a 19-inch monitor the paddle was 4 inches. The period between successive targets appearances was 3 seconds.

Thus, for one test session of 2 minutes, 40 player movements were made one-half in each direction (left-right or up-down). One-half of the movements were medium amplitude (the paddle moved one-third to one-half the distance of the monitor dimensions), and one-half were large amplitude (the paddle moved one-half to full monitor dimensions). The locations of target appearance were random on the screen. 
Development of a Computerized assessment tool for hand-arm function after stroke: test-retest reliability and convergent validity

A. Participant manipulating a doorknob with mouse attached on it.

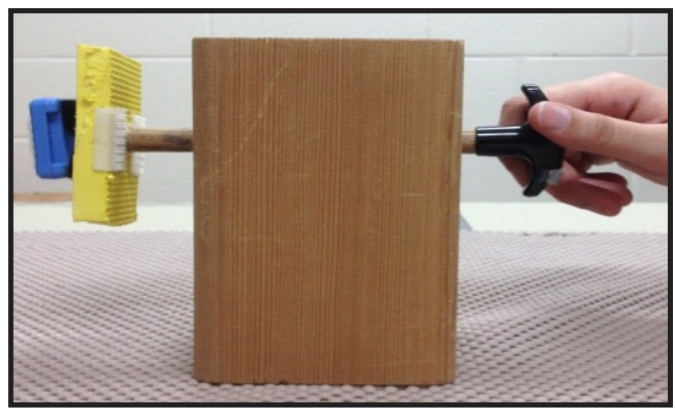

C. Trajectories of object manipulation

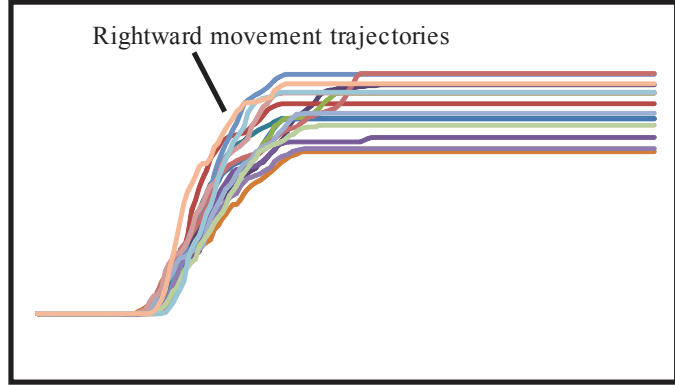

\section{B. Components of game task}

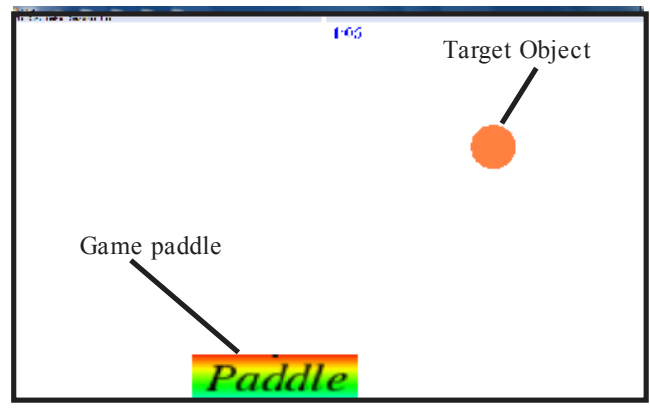

D. Mean outcomes of a game task.

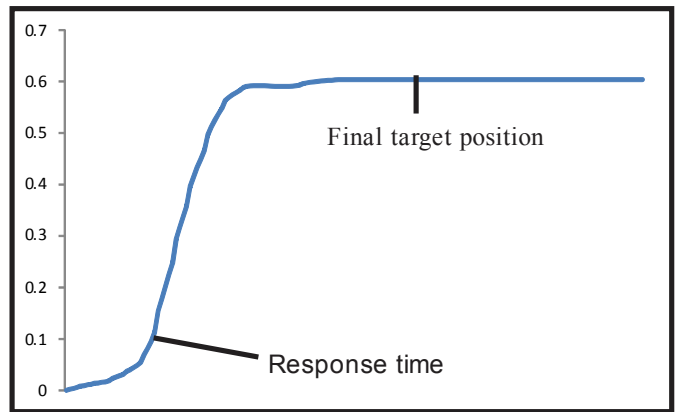

Panel A Doorknob manipulation, Panel B presents the components of a game task where paddle is controlled by user object manipulation and circle is the computer target. Panel C presents sorted game events of rightward trajectories of object manipulation task. Panel D presents mean outcome of game task, the response time and final target position.

Figure 4- Motor skill Game task and its components. 
Development of a Computerized assessment tool for hand-arm function after stroke: test-retest reliability and convergent validity

\subsection{DATA ANALYSES}

\section{Cyclic tracking task performance measures}

Figure 3-C presents synchronous plots of the target motion (circle) and object manipulation (square) performed by a client for a typical GHA Cyclic tracking task. The quality of the movement is quantified by computing TRE, which is the sum of the difference in position between the computer reference target and the participant position. $\mathrm{AC}$ is the variation in movement amplitude for each half cycle, i.e. in 20 cycles there would be 20 left to right movement and 20 right to left movement or 20 top to bottom or 20 bottom to top. The amplitude consistency was then determined as standard deviations normalized by the mean for all the cycles over $60 \mathrm{~s}$. For a detailed description of this module analysis, see (53), (36).

\section{Motor Skill tasks Performance Measures}

This module records the time of each target object appearance and disappearance and logs the position coordinates of target objects and game paddle at a rate of $80 \mathrm{~Hz}$. Figure $4 \mathrm{C}$ presents the overlay trajectories of participant manipulating movements for all rightward game events recorded in one game session, and figure 4D shows the average of all the game events. The following variables averaged over all rightward game movements were determined; (a) movement onset time (MOT) i.e., the time from target appearance to start the game sprite (paddle) movement and (b) the game success rate (SR) i.e the percentage of target objects that were caught, and (c) Movement Variation (MV) i.e. the standard deviation calculated from the mean of all movement trajectories. For a detailed description of the analysis of the game module refer to previous 
Development of a Computerized assessment tool for hand-arm function after stroke: test-retest reliability and convergent validity

publications (54), (55), (56). MATLAB (The Math Works, Natick, MA, version 2010a) was used to compute the outcome measures for the both Cyclic tracking and Motor skill game tasks.

\subsection{PROTOCOL}

The first session included administration of the WMFT followed by GHA assessment. The WMFT has 15 time-based tasks (44). In the first session, 8 of the 15 tasks were performed. Before administration, each task was explained to the participants. 120 seconds were given to complete each task but at the same time, the participants were asked to complete the tasks as soon as possible. Tasks which were unable to be performed were given a score of 120 seconds. Also, every task was graded on an ordinal scale of 0 to 5 , where 0 indicated did not perform at all, and 5 indicated performed with normal movement. The GHA assessment was administered after the WMFT. The participants were comfortably seated, and an adjustable table where objects were placed and manipulated was put in the front. A computer monitor was placed 1.5 meters in front of the participant at eye level. All the participants were provided with a demonstration of testing. After one practice trial, each task was recorded. Three to four days later the second test was performed and the remaining 7 tasks of WMFT were completed, followed by grip strength testing of the most affected hand. Grip strength was tested using an isometric hand dynamometer (G100, Biometrics Ltd., UK) in the testing position recommended by the American Society of Hand Therapists. The average score of two consecutive trials was used for the analysis. All GHA tasks were again tested in the same manner as tested in the first session. For the between group comparison of GHA performance measures, 20 healthy subjects performed the same tasks in one session and 
Development of a Computerized assessment tool for hand-arm function after stroke: test-retest reliability and convergent validity

their performances were compared with performances of 20 randomly chosen stroke participants. 
Development of a Computerized assessment tool for hand-arm function after stroke: test-retest reliability and convergent validity

\subsection{STATISTICAL ANALYSES}

ICC values were calculated to determine the relative test-retest reliability (57), (58). ICC values were interpreted as very high ( ICC >0.9), high (ICC > 0.7), moderate (ICC between $0.5-0.7)$ and low (ICC < 0.5) (59). Absolute reliability was calculated using

SEM, using the formula- $E M=S D \times(\sqrt{1-I C C)}$ where SD is the average standard deviation of the two session scores (60), (61). A paired student ' $t$ ' test for mean differences between the two sessions score was also done. MDC was calculated using the formula- $M D C=1.65 \times S E M \times \sqrt{2}$, where 1.65 is 2 -tailed tabled $z$-value for $90 \%$ confidence interval and $\sqrt{2}$ is the variance of 2 measurements (62), (63). MDC can be interpreted as the magnitude of change below which there is more than a $90 \%$ chance that no real change has occurred. Thus, any change greater than the MDC signifies a real or a significant change.

Convergent validity was analyzed using Pearson correlation coefficient between GHA task performance measures and WMFT (time) and grip strength. Spearman rank correlation coefficient (rho) to determine the strength of relationship between GHA task performance measures and WMFT (movement quality) was also calculated. The strength of the correlation was interpreted as high (r/rho $>0.7)$, moderate $(0.4$ to 0.7$)$ and low $(<$ 0.4) (10), (64). A within stroke group correlation of GHA performance measures was done by computing a Pearson correlation coefficient. Known group validity was evaluated by performing a between group analysis for stroke and healthy participants, and was done by using paired Student's t test. Data was analyzed with IBM SPSS 
Development of a Computerized assessment tool for hand-arm function after stroke: test-retest reliability and convergent validity

Statistics for Windows, Version 19.0. Armonk, NY: IBM Corp. Statistical significance was $\mathrm{p}<0.05$ (two-tailed). 
Development of a Computerized assessment tool for hand-arm function after stroke: test-retest reliability and convergent validity

\subsection{RESULTS}

\section{Demographics}

Table 2 presents the demographic characteristics of study participants. 30 participants 20 males and 10 females, with a mean age of 68.41 years old with a history of single stroke within previous six to twenty-four months were recruited. Mean values for WMFT (time) were 282.21 seconds, and WMFT (functional ability) was 45.2.

Table 2-Clinical and demographic characteristics of study participants.

\begin{tabular}{|ll|}
\hline Number of Participants & 30 \\
Men/Women & $20 / 10$ \\
Mean age (year) & 68.41 \\
Mean time since stroke (year) & 1.32 \\
Mean (SD) of WMFT (time) & $282.21(295.13)$ \\
Mean (SD) of WMFT (functional ability) & $45.2(12.35)$ \\
Type of stroke: & \\
i. $\quad$ Ischemic & 25 \\
ii. Hemorrhagic $\quad 5$ \\
\hline
\end{tabular}


Development of a Computerized assessment tool for hand-arm function after stroke: test-retest reliability and convergent validity

\subsubsection{Raw data of the GHA assessment outcomes and their key features}

\subsubsection{Cyclic tracking}

Figure 5 presents the typical plots of participant motion trajectories during the cyclic tracking task for each of the 5 object manipulation tasks. On the left side are the objects that were manipulated for each task, in the middle are the trajectories of a healthy subject and on the right are the trajectories of a stroke subject. The blue dotted lines represent the amplitude of the reference wave.

The trajectories of a healthy client when compared with that of stroke client, clearly indicate poor movement consistency in the latter. Each sinusoid wave i.e. one peak and one trough represents one full cyclic movement or manipulation of the object. For example, while manipulating a mug, the peak would be pronation and trough would be supination. Both peaks and troughs are over or undershooting the reference trajectory range in the stroke client, which represents poor coordination and movement control. On the other hand for the healthy subject, they are quite consistent. For the doorknob trajectory, there is most overshooting of the peaks, which shows that small fine movements had the poorest control among all tasks. Also, a couple of missed cycles are seen in the stroke trajectories, which does not occur in the healthy participants. Lastly, in the stroke trajectories sudden jerky movements are observed, which for the healthy participants are smooth and consistent. These findings of the raw data clearly present the outcome characteristics we expected from the healthy and stroke group performances. 
Development of a Computerized assessment tool for hand-arm function after stroke: test-retest reliability and convergent validity

Group 1 (healthy)

Objects manipulated during the tasks

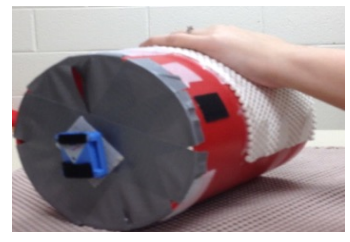

1. Cylinder

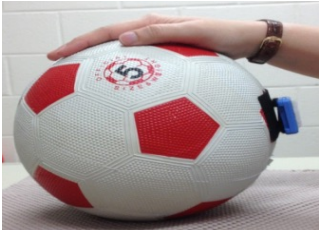

2. Soccer Ball

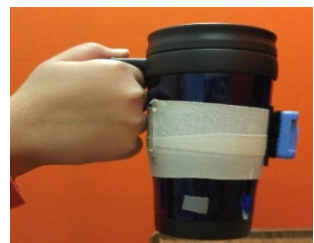

3. Mug

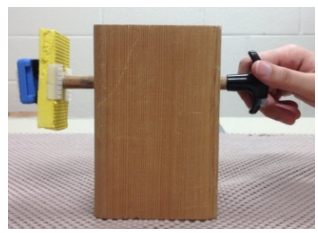

4. Doorknob

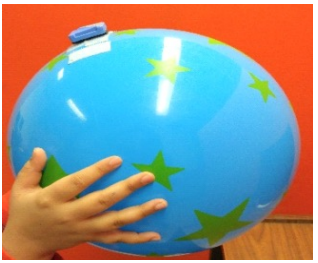

5. Bilateral
5 tracking tasks representing movement amplitude, and consistency.

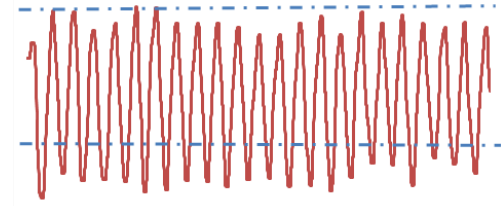

Group 2 (stroke)

5 tracking tasks presenting movement amplitude, and consistency.
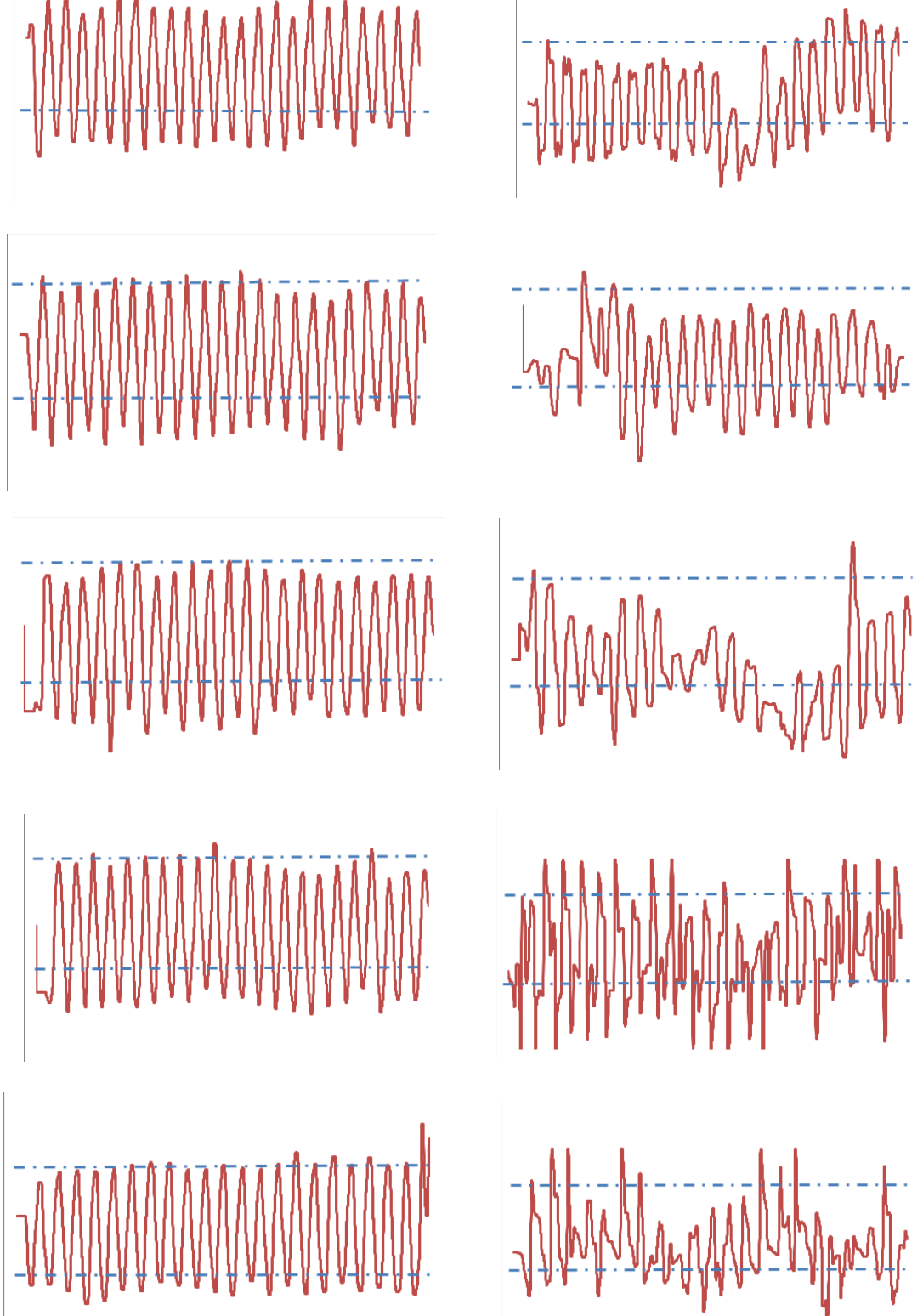

Figure 5- User motion trajectories of the Cyclic tracking tasks of normal and stroke group. Two groups, stroke and healthy performed the similar 5 GHA cyclic tracking tasks. On the left are the 5 objects that were manipulated during the tasks, in the middle are performances of a healthy participant and on the extreme right are the performances of a stroke subject. The blue dotted line represents the amplitude of the reference trajectory. On the $\mathrm{X}$-axis is the time in seconds and on the $\mathrm{Y}$-axis is the movement amplitude. 
Development of a Computerized assessment tool for hand-arm function after stroke: test-retest reliability and convergent validity

\subsubsection{Motor skill Game}

Figure 6 presents the overlay typical plots of the participant motion trajectories during the Motor skill game tasks for each of the 5 object manipulation tasks. On the left side are the objects that were manipulated during each task, in the middle are the trajectories of a healthy client, and on the right are the trajectories of a stroke client.

The plots of normal and stroke clients had a huge difference on observation. Each trajectory represents one game event. Firstly, the trajectories of game events are not consistent with each other and are quite different from each other in stroke performances, whereas for the healthy subject they appear smooth and consistent. Secondly, MOT is delayed and extended among stroke trajectories and is early and fast for healthy, which shows that the stroke participant took more time to initiate a movement than the healthy participants. Thirdly, the over and undershooting of amplitude among trajectories for the stroke participants represents jerky movements made during the task performances which are not present in the healthy performances. Lastly, some straight trajectories are seen in the stroke performances, which mean those game events were not entertained during the task. Nothing likewise was seen among healthy performances. 
Development of a Computerized assessment tool for hand-arm function after stroke: test-retest reliability and convergent validity

Objects manipulated during the tasks

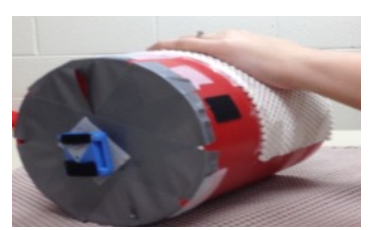

1. Cylinder

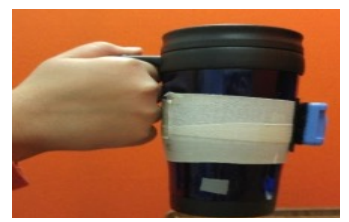

2. Mug

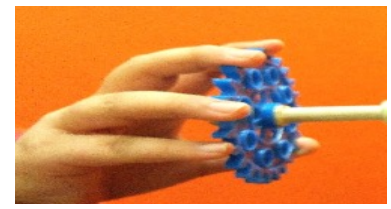

3. Jar lid

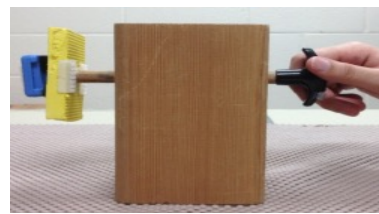

4. Doorknob

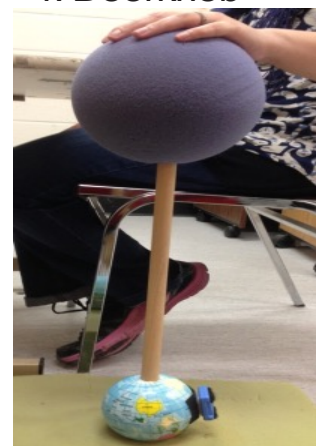

5. Jovstick
Group 2 (healthy) outcome of 5 manipulation tasks. Game events are

Group 1 (stroke) outcome of 5 manipulation tasks. Game events are not consistent and variation is low. consistent and variation is high.
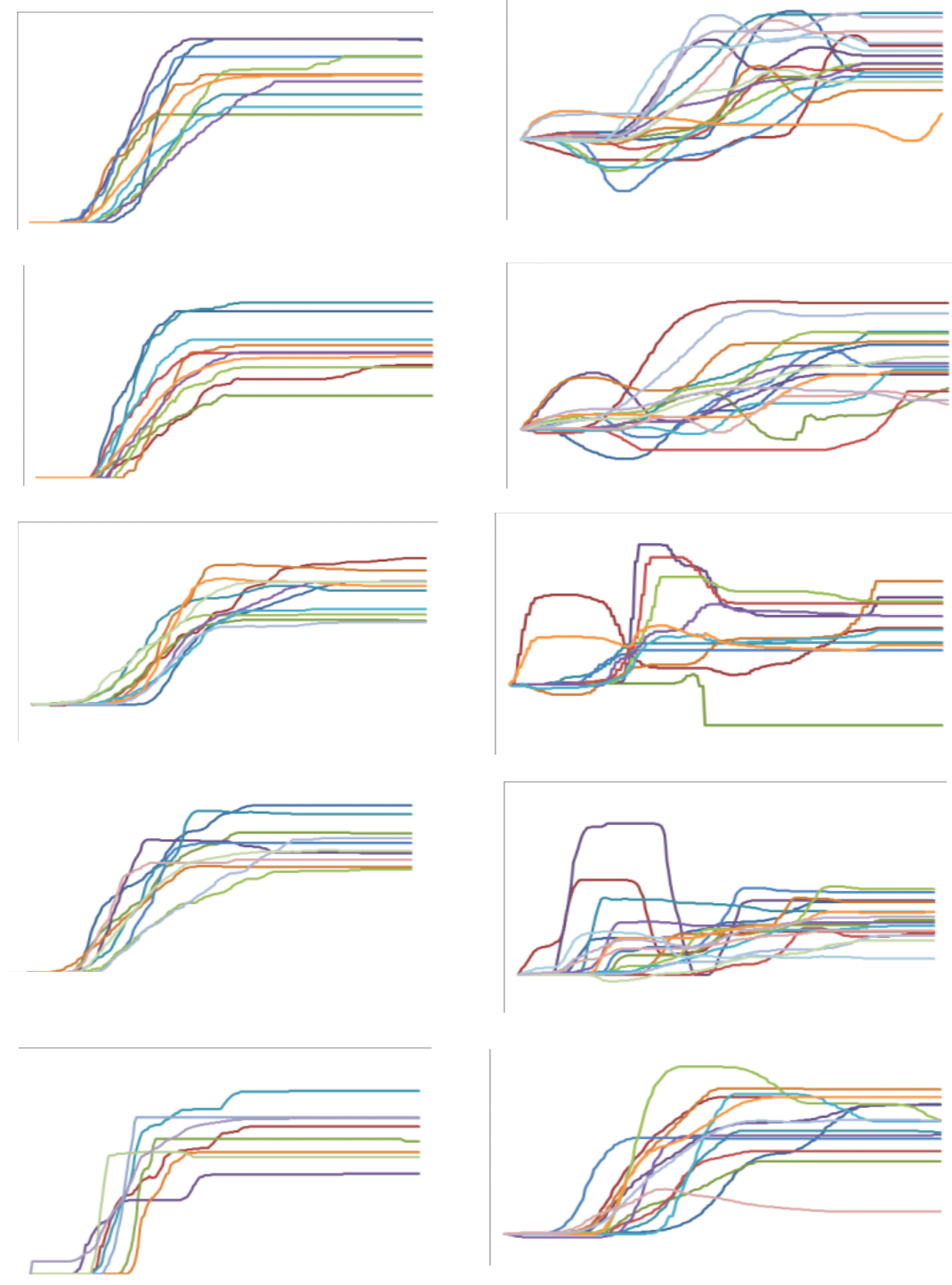

Figure 6- User motion trajectories of Motor skill game tasks of normal and stroke group. Two groups, stroke and healthy performed the similar 5 GHA motor skill game tasks. On the left are the 5 objects that were manipulated during the tasks, in the middle are performances of a healthy participant and on the extreme right are the performances of a stroke subject. On the $\mathrm{X}$-axis is the time in seconds and on the Y-axis is the movement amplitude. 
Development of a Computerized assessment tool for hand-arm function after stroke: test-retest reliability and convergent validity

\subsubsection{Test-Retest reliability}

Table 3 presents the results of statistical analysis of relative and absolute reliability of the TRE of the Cyclic tracking tasks. Test-retest reliability was high $(\mathrm{ICC}>0.7)$ for the cylinder, mug, and soccer ball, moderate (ICC between 0.5 and 0.7 ) for the doorknob, and low $(\mathrm{ICC}<0.5)$ for the bilateral task. SEM as a percentage of the group mean value ranged from $6.96 \%$ to $23.78 \%$ and was less than $15 \%$ in the majority of cases. MDC as a percentage of group mean value ranged from $19.16 \%$ to $55.55 \%$ and was less than $30 \%$ in the majority of cases. Paired Student's t tests showed no significant differences in mean performance measures for each task between the sessions $(p>0.05)$.

Table 3- Test-retest reliability of TRE outcome measures. The table presents ICC values (95\%CI), Mean (SD), SEMs, MDCs, and t-statistics.

\begin{tabular}{|c|c|c|c|c|c|c|}
\hline \multirow[t]{2}{*}{ Object } & \multirow{2}{*}{$\begin{array}{l}\text { ICC }(95 \% \\
\text { CI) }\end{array}$} & \multicolumn{2}{|c|}{ Mean (SD) } & \multirow{2}{*}{$\begin{array}{l}\text { SEM } \\
\text { (\% of } \\
\text { mean) }\end{array}$} & \multirow{2}{*}{$\begin{array}{l}\text { MDC } \\
\text { (\%of } \\
\text { mean) }\end{array}$} & \multirow{2}{*}{$\begin{array}{l}\text { t-test } \\
(p- \\
\text { value) }\end{array}$} \\
\hline & & Test 1 & Test 2 & & & \\
\hline Cylinder & $\begin{array}{l}0.81(0.61- \\
0.92)\end{array}$ & $751.08(173.30)$ & 769.03(206.36) & $\begin{array}{l}75.54 \\
(10.06)\end{array}$ & $\begin{array}{l}176.02 \\
(23.43)\end{array}$ & 0.56 \\
\hline $\begin{array}{l}\text { Soccer } \\
\text { Ball }\end{array}$ & $\begin{array}{l}0.72(0.36- \\
0.88)\end{array}$ & $840.30(200.60)$ & $823.0(197.65)$ & $\begin{array}{l}106.15 \\
(12.64)\end{array}$ & $\begin{array}{l}247.32 \\
(29.44)\end{array}$ & 0.65 \\
\hline Mug & $\begin{array}{l}0.87(0.71- \\
0.94)\end{array}$ & $814.89(185.91)$ & 799.95(209.12) & $\begin{array}{l}67.03 \\
(8.23)\end{array}$ & $\begin{array}{l}156.18 \\
(19.16)\end{array}$ & 0.57 \\
\hline $\begin{array}{l}\text { Door } \\
\text { knob }\end{array}$ & $\begin{array}{l}0.62(0.10- \\
0.84)\end{array}$ & 757.03(134.09) & $760.98(258.41)$ & $\begin{array}{l}82.66 \\
(6.96)\end{array}$ & $\begin{array}{l}192.59 \\
(25.44)\end{array}$ & 0.94 \\
\hline $\begin{array}{l}\text { Bilateral } \\
\text { Task }\end{array}$ & $\begin{array}{l}0.44(0.13- \\
0.73)\end{array}$ & $658.31(209.75)$ & $734.24(197.53)$ & $\begin{array}{l}156.95 \\
(23.78)\end{array}$ & $\begin{array}{l}365.7 \\
(55.55)\end{array}$ & 0.09 \\
\hline
\end{tabular}


Development of a Computerized assessment tool for hand-arm function after stroke: test-retest reliability and convergent validity

Table 4 presents the results of the statistical analysis of relative and absolute reliability for the AC of the cyclic tracking task. Test-retest reliability was high (ICC > 0.7) for cylinder and doorknob, moderate (ICC between 0.5 and 0.7 ) for the soccer ball and bilateral task, and low (ICC < 0.5) for the mug. SEM as a percentage of the group mean values ranged from $3.11 \%$ to $30.33 \%$ and was less than $20 \%$ in the majority of cases. MDC as a percentage of group mean value ranged from $7.22 \%$ to $84.07 \%$ and was less than $40 \%$ in the majority of cases. Paired Student's t tests showed no significant differences in mean performance measures for each task between the sessions $(p>0.05)$.

Table 4-Test-retest reliability of AC outcome measure. The table presents ICC (95\%CI), Mean (SD), SEMs, MDCs, and t-statistics.

\begin{tabular}{|c|c|c|c|c|c|c|}
\hline \multirow[t]{2}{*}{ Object } & \multirow[t]{2}{*}{ ICC (95\% CI) } & \multicolumn{2}{|c|}{ Mean (SD) } & \multirow{2}{*}{$\begin{array}{l}\text { SEM }(\% \\
\text { of mean) }\end{array}$} & \multirow{2}{*}{$\begin{array}{l}\text { MDC } \\
\text { (\% of } \\
\text { mean) }\end{array}$} & \multirow{2}{*}{$\begin{array}{l}\text { t-test (p- } \\
\text { value) }\end{array}$} \\
\hline & & Test 1 & Test 2 & & & \\
\hline Cylinder & $\begin{array}{l}0.76(0.46- \\
0.89)\end{array}$ & $22.66(7.03)$ & $23.27(7.8)$ & $\begin{array}{l}3.44 \\
(15.18)\end{array}$ & $\begin{array}{l}8.02 \\
(35.39)\end{array}$ & 0.65 \\
\hline $\begin{array}{l}\text { Soccer } \\
\text { Ball }\end{array}$ & $0.51(0.13-0.75)$ & $28.27(12.25)$ & $30.3(12.16)$ & $\begin{array}{l}8.58 \\
(30.33)\end{array}$ & $\begin{array}{l}23.76 \\
(84.07)\end{array}$ & 0.61 \\
\hline Mug & $\begin{array}{l}0.28(0.69- \\
0.69)\end{array}$ & $26.29(5.74)$ & $28.35(8.58)$ & $\begin{array}{l}4.87 \\
(18.52)\end{array}$ & $\begin{array}{l}11.35 \\
(43.17)\end{array}$ & 0.31 \\
\hline $\begin{array}{l}\text { Door } \\
\text { Knob }\end{array}$ & $\begin{array}{l}0.71(0.31- \\
0.85)\end{array}$ & $26.59(8.38)$ & $25.66(7.55)$ & $\begin{array}{l}4.51 \\
(16.96)\end{array}$ & $\begin{array}{l}10.51 \\
(39.52)\end{array}$ & 0.54 \\
\hline $\begin{array}{l}\text { Bilateral } \\
\text { task }\end{array}$ & $\begin{array}{l}0.67(0.31- \\
0.85)\end{array}$ & $23.35(8.94)$ & $25.77(9.2)$ & $\begin{array}{l}5.14 \\
(21.12)\end{array}$ & $\begin{array}{l}11.96 \\
(51.22)\end{array}$ & 0.16 \\
\hline
\end{tabular}


Development of a Computerized assessment tool for hand-arm function after stroke: test-retest reliability and convergent validity

Table 5 presents the results of the statistical analysis of relative and absolute reliability for SR of the Motor skill game task. Test-retest reliability was high (ICC > 0.7) for jar lid, moderate (ICC between 0.5 and 0.7 ) for cylinder, mug, joystick, and low (ICC $<0.5$ ) for doorknob. SEM as a percentage of the group mean values ranged from $5.28 \%$ to $7.39 \%$ and was less than $10 \%$ in all of the cases. MDC as a percentage of group mean value ranged from $12.31 \%$ to $17.93 \%$ and was less than $20 \%$ in all of the cases. Paired Student's t tests showed no significant differences in mean performance measures for each task between the sessions $(\mathrm{p}>0.05)$.

Table 5- Test-retest reliability of SR outcome measure. The table presents ICC (95\%CI), Mean (SD), SEMs, MDCs, t-statistics.

\begin{tabular}{|c|c|c|c|c|c|c|}
\hline \multirow[t]{2}{*}{ Object } & \multirow{2}{*}{$\begin{array}{l}\text { ICC }(95 \% \\
\text { CI) }\end{array}$} & \multicolumn{2}{|c|}{ Mean (SD) } & \multirow{2}{*}{$\begin{array}{l}\text { SEM } \\
(\% \text { of } \\
\text { mean })\end{array}$} & \multirow[t]{2}{*}{ MDC } & \multirow{2}{*}{$\begin{array}{l}\text { t-test } \\
\text { (p- } \\
\text { value) }\end{array}$} \\
\hline & & Test 1 & Test 2 & & & \\
\hline Cylinder & $\begin{array}{l}0.52(0.02- \\
0.77)\end{array}$ & $94.73(10.53)$ & $95.61(8.15)$ & $\begin{array}{l}7.29 \\
(7.7)\end{array}$ & $\begin{array}{l}16.99 \\
(17.93\end{array}$ & 0.66 \\
\hline Mug & $\begin{array}{l}0.51(0.15- \\
0.76)\end{array}$ & $93.60(7.07)$ & $91.01(15.07)$ & $\begin{array}{l}4.94 \\
(5.28)\end{array}$ & $\begin{array}{l}11.53 \\
(12.31 \\
)\end{array}$ & 0.34 \\
\hline $\begin{array}{l}\text { Door } \\
\text { knob }\end{array}$ & $\begin{array}{l}0.45(0.14- \\
0.74)\end{array}$ & $94.80(9.45)$ & $87.93(20.25)$ & $\begin{array}{l}7.01 \\
(7.39)\end{array}$ & $\begin{array}{l}16.32 \\
(17.21 \\
)\end{array}$ & 0.06 \\
\hline Jar lid & $\begin{array}{l}0.82(0.61- \\
0.91)\end{array}$ & $89.02(14.71)$ & $90.19(14.12)$ & $\begin{array}{l}6.24 \\
(7.01)\end{array}$ & $\begin{array}{l}14.54 \\
(16.33 \\
)\end{array}$ & 0.58 \\
\hline Joystick & $\begin{array}{l}0.56(0.03- \\
0.81)\end{array}$ & $93.47(8.21)$ & $93.77(6.15)$ & $\begin{array}{l}5.45 \\
(5.83)\end{array}$ & $\begin{array}{l}12.69 \\
(13.57 \\
)\end{array}$ & 0.85 \\
\hline
\end{tabular}


Development of a Computerized assessment tool for hand-arm function after stroke: test-retest reliability and convergent validity

Table 6 presents the results of the statistical analysis of relative and absolute reliability for MOT of the Motor skill game task. Test-retest reliability was high (ICC $>0.7$ ) for the doorknob, moderate (ICC between 0.5 and 0.7 ) for the mug, jar lid, and low $($ ICC $<0.5)$ for cylinder and joystick. SEM as a percentage of the group mean values ranged from $10.84 \%$ to $18.88 \%$ and was less than $15 \%$ in the majority of cases. MDC as a percentage of group mean value ranged from $26.50 \%$ to $43.33 \%$ and was less than $30 \%$ in the majority of cases. Paired Student $t$ tests showed no significant differences in mean performance measures for each task between the sessions $(\mathrm{p}>0.05)$.

Table 6- Test-retest reliability of MOT outcome measure. The table presents ICC (95\%CI), Mean (SD), MDCs and t-statistics.

\begin{tabular}{|c|c|c|c|c|c|c|}
\hline \multirow[t]{2}{*}{ Object } & \multirow{2}{*}{$\begin{array}{l}\text { ICC }(95 \% \\
\text { CI) }\end{array}$} & \multicolumn{2}{|c|}{ Mean (SD) } & \multirow{2}{*}{$\begin{array}{l}\text { SEM } \\
\text { (\% of } \\
\text { mean) }\end{array}$} & \multirow{2}{*}{$\begin{array}{l}\text { MDC } \\
(\% \text { of } \\
\text { mean })\end{array}$} & \multirow{2}{*}{$\begin{array}{l}\text { t-test } \\
\text { (p- } \\
\text { value) }\end{array}$} \\
\hline & & Test 1 & Test 2 & & & \\
\hline Cylinder & $\begin{array}{l}0.45(0.37- \\
0.76)\end{array}$ & $0.83(0.13)$ & $0.82(0.14)$ & $\begin{array}{l}0.09 \\
(10.84)\end{array}$ & $\begin{array}{l}0.22 \\
(26.50)\end{array}$ & 0.69 \\
\hline Mug & $\begin{array}{l}0.51(0.11- \\
0.78)\end{array}$ & $0.77(0.14)$ & $0.74(0.17)$ & $\begin{array}{l}0.1 \\
(12.98)\end{array}$ & $\begin{array}{l}0.23 \\
(29.87)\end{array}$ & 0.45 \\
\hline Door knob & $\begin{array}{l}0.71(0.32- \\
0.86)\end{array}$ & $0.77(0.19)$ & $0.78(0.19)$ & $\begin{array}{l}0.1 \\
(12.98)\end{array}$ & $\begin{array}{l}0.24 \\
(31.16)\end{array}$ & 0.79 \\
\hline Jar lid & $\begin{array}{l}0.62(0.14- \\
0.83)\end{array}$ & $0.75(0.14)$ & $0.73(0.18)$ & $\begin{array}{l}0.09 \\
(12.0)\end{array}$ & $\begin{array}{l}0.2 \\
(26.66)\end{array}$ & 0.48 \\
\hline Joystick & $\begin{array}{l}0.41(0.22- \\
0.71)\end{array}$ & $0.90(0.22)$ & $0.82(0.19)$ & $\begin{array}{l}0.17 \\
(18.88)\end{array}$ & $\begin{array}{l}0.39 \\
(43.33)\end{array}$ & 0.12 \\
\hline
\end{tabular}


Development of a Computerized assessment tool for hand-arm function after stroke: test-retest reliability and convergent validity

Table 7 presents the results of statistical analysis of relative and absolute reliability for MV obtained from the Motor skill game task. Test-retest reliability was moderate (ICC between 0.5 and 0.7$)$ for the doorknob, and low $(\mathrm{ICC}<0.5)$ for the cylinder, mug, jar lid and joystick. The standard error of measure (SEM) as a percentage of the group mean values ranged from $13.04 \%$ to $35.71 \%$ and was less than $30 \%$ in the majority of the cases. The minimal detectable change (MDC) as a percentage of group mean value ranged from $46.15 \%$ to $86.17 \%$ and was less than $50 \%$ in two of the five cases. Paired Student's t tests showed no significant differences in mean performance measures for each task between the sessions $(\mathrm{p}>0.05)$.

Table 7- Test-retest reliability of MV outcome measure. The table presents ICC (95\%CI), Mean, (SD), SEMs, MDCs, and t-statistics.

\begin{tabular}{|c|c|c|c|c|c|c|}
\hline \multirow[t]{2}{*}{ Object } & \multirow{2}{*}{$\begin{array}{l}\text { ICC }(95 \% \\
\text { CI) }\end{array}$} & \multicolumn{2}{|c|}{ Mean (SD) } & \multirow{2}{*}{$\begin{array}{l}\text { SEM } \\
\text { (\% of } \\
\text { mean) }\end{array}$} & \multirow{2}{*}{$\begin{array}{l}\text { MDC } \\
\text { (\% of } \\
\text { mean) }\end{array}$} & \multirow{2}{*}{$\begin{array}{l}\text { t-test } \\
\text { (p- } \\
\text { value) }\end{array}$} \\
\hline & & Test 1 & Test 2 & & & \\
\hline Cylinder & $\begin{array}{l}0.22(0.61- \\
0.62)\end{array}$ & $0.14(0.06)$ & $0.12(0.06)$ & $\begin{array}{l}0.05 \\
(35.71)\end{array}$ & $\begin{array}{l}0.12 \\
(85.71)\end{array}$ & 0.23 \\
\hline Mug & $\begin{array}{l}0.31(0.66- \\
0.70)\end{array}$ & $0.13(0.03)$ & $0.13(0.03)$ & $\begin{array}{l}0.03 \\
(23.04)\end{array}$ & $\begin{array}{l}0.06 \\
(46.15)\end{array}$ & 0.61 \\
\hline Door knob & $\begin{array}{l}0.68(0.27- \\
0.86)\end{array}$ & $0.13(0.05)$ & $0.14(0.05)$ & $\begin{array}{l}0.03 \\
(23.07)\end{array}$ & $\begin{array}{l}0.06 \\
(46.15)\end{array}$ & 0.58 \\
\hline Jar lid & $\begin{array}{l}0.47(0.50- \\
0.56)\end{array}$ & $0.15(0.06)$ & $0.11(0.06)$ & $\begin{array}{l}0.04 \\
(26.67)\end{array}$ & $\begin{array}{l}0.1 \\
(66.66)\end{array}$ & 0.11 \\
\hline Joystick & $\begin{array}{l}0.23(0.62- \\
0.74)\end{array}$ & $0.15(0.05)$ & $0.14(0.04)$ & $\begin{array}{l}0.05 \\
(33.33)\end{array}$ & $\begin{array}{l}0.11 \\
(78.57)\end{array}$ & 0.74 \\
\hline
\end{tabular}




\subsubsection{Convergent validity}

A correlation analysis between GHA outcome measures and WMFT (time and movement quality) and grip strength was performed. The Pearson correlation coefficient was calculated between the WMFT (time), grip strength and GHA outcome measuresTRE for the Cyclic tracking task and MOT and SR for the Motor skill game task. The Spearman correlation coefficient was calculated for WMFT (movement quality) and GHA outcome measures for the 5 cyclic tracking and 5 Motor skill game tasks.

Only 2 out of 15 correlations between GHA cyclic tracking performance outcome (TRE) and scores of WMFT were significant. Low correlations were observed between WMFT (movement quality) and TRE of bilateral task (rho $=0.36, \mathrm{p}=0.011)$ and between grip strength and TRE of soccer ball $(r=0.48, p=0.008)$.

For the Motor skill game task only 6 out of 30 correlations between GHA performance scores (MOT and SR) and WMFT were significant. Moderate correlations were seen between grip strength and SR of doorknob $(r=0.53, p=0.003)$, grip strength and MOT of doorknob $(\mathrm{r}=0.53, \mathrm{p}=0.004)$. Low correlations were seen between WMFT (time) with SR of doorknob $(r=0.38, p=0.037)$, WMFT (movement quality) and MOT of cylinder ( $\mathrm{rho}=0.41, \mathrm{p}=0.034)$, WMFT (movement quality) with SR of doorknob (rho $=0.41, \mathrm{p}=0.037)$, Grip strength and MOT of jar lid $(\mathrm{r}=0.47, \mathrm{p}=0.010)$. 
Development of a Computerized assessment tool for hand-arm function after stroke: test-retest reliability and convergent validity

\subsubsection{Correlation of tasks within stroke group}

A Pearson correlation analysis was conducted to determine if a significant correlation existed in TRE performance scores among 5 different object manipulation tasks. With one exception, there were no significant correlations in the performance scores among the cyclic tracking tasks. The one exception was a high correlation $(\mathrm{r}=$ $0.72, \mathrm{p}=0.001$ ) between the cylinder and soccer ball tasks.

Further, a Pearson correlation analysis was conducted to determine if a significant correlation existed in SR performance scores among 5 different object manipulation tasks. With two exceptions there were no significant correlations in the performance scores of the Motor skill game tasks. The two exceptions were low correlations between cylinder and doorknob $(\mathrm{r}=0.39, \mathrm{p}=0.035)$, and joystick and doorknob $(\mathrm{r}=0.37, \mathrm{p}=$ $0.042)$ tasks. 
Development of a Computerized assessment tool for hand-arm function after stroke: test-retest reliability and convergent validity

\subsubsection{Known group validity/comparison of tasks between stroke and normal healthy group}

Figure 7, 8, and 9 present between group comparison of the means and SEMs of GHA Cyclic tracking (TRE), and Motor skill game (MOT and MV) outcome measures of 5 object manipulation tasks. Table 8,9 and 10 presents the results of the statistical analysis comparing the performance scores between the two groups. The performance scores for both the Cyclic tracking and Motor skill game task were significantly better in the control group. There was one exception where MV of jar lid task had $p$-value $=0.15$. 
Development of a Computerized assessment tool for hand-arm function after stroke: test-retest reliability and convergent validity

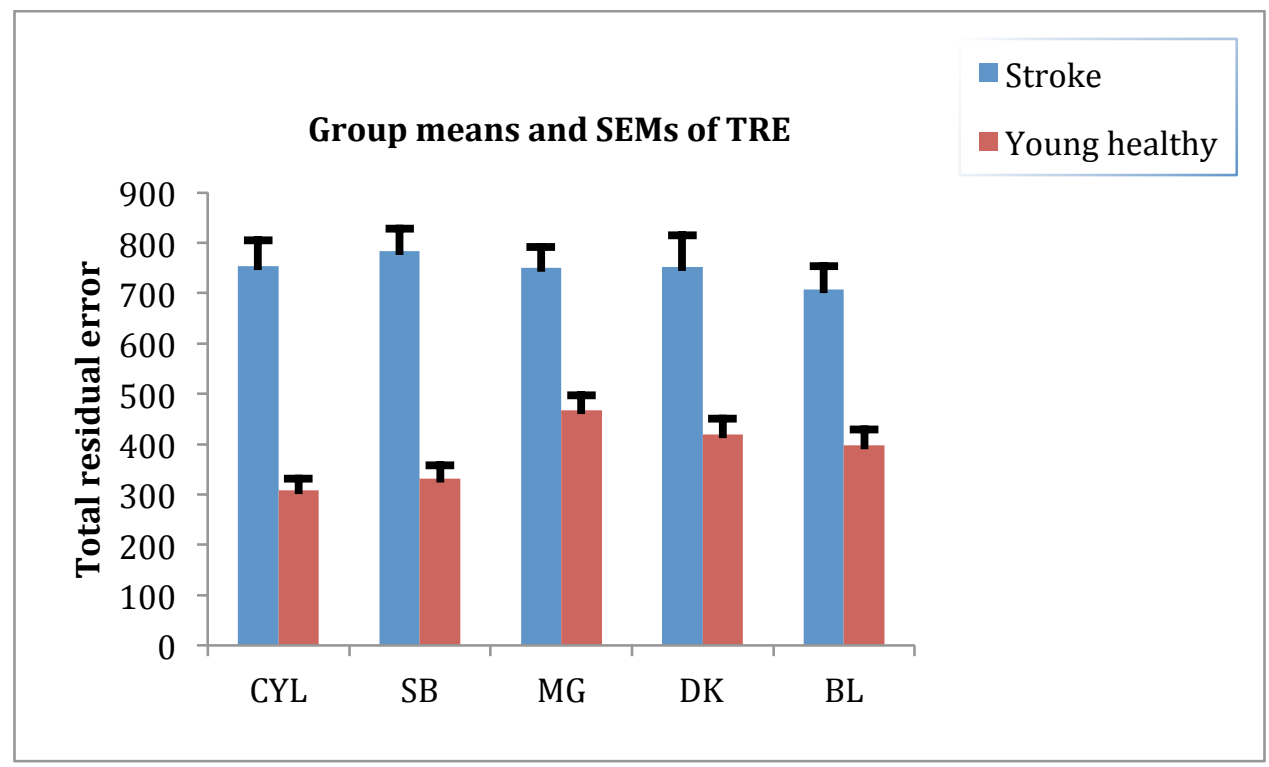

Figure 7- Between-group comparisons of TRE. Blue bars represent the performance of stroke group, and red bars represent the performance of the healthy group. On the $\mathrm{X}$-axis are the five objects that were manipulated during the tasks and on the $\mathrm{Y}$ axis is the TRE outcome in pixels. The error caps represent the standard error of the mean for both groups.

Table 8- between group statistical analyses of TRE.

\begin{tabular}{|lll|}
\hline Total residual error & t statistics $(\mathbf{d f}=\mathbf{3 8})$ & Sig. (2-tailed) \\
CYL & 7.82 & .000 \\
SB & 8.73 & .000 \\
MG & 5.52 & .000 \\
DK & 4.67 & .000 \\
BL & 5.57 & .000 \\
\hline
\end{tabular}


Development of a Computerized assessment tool for hand-arm function after stroke: test-retest reliability and convergent validity

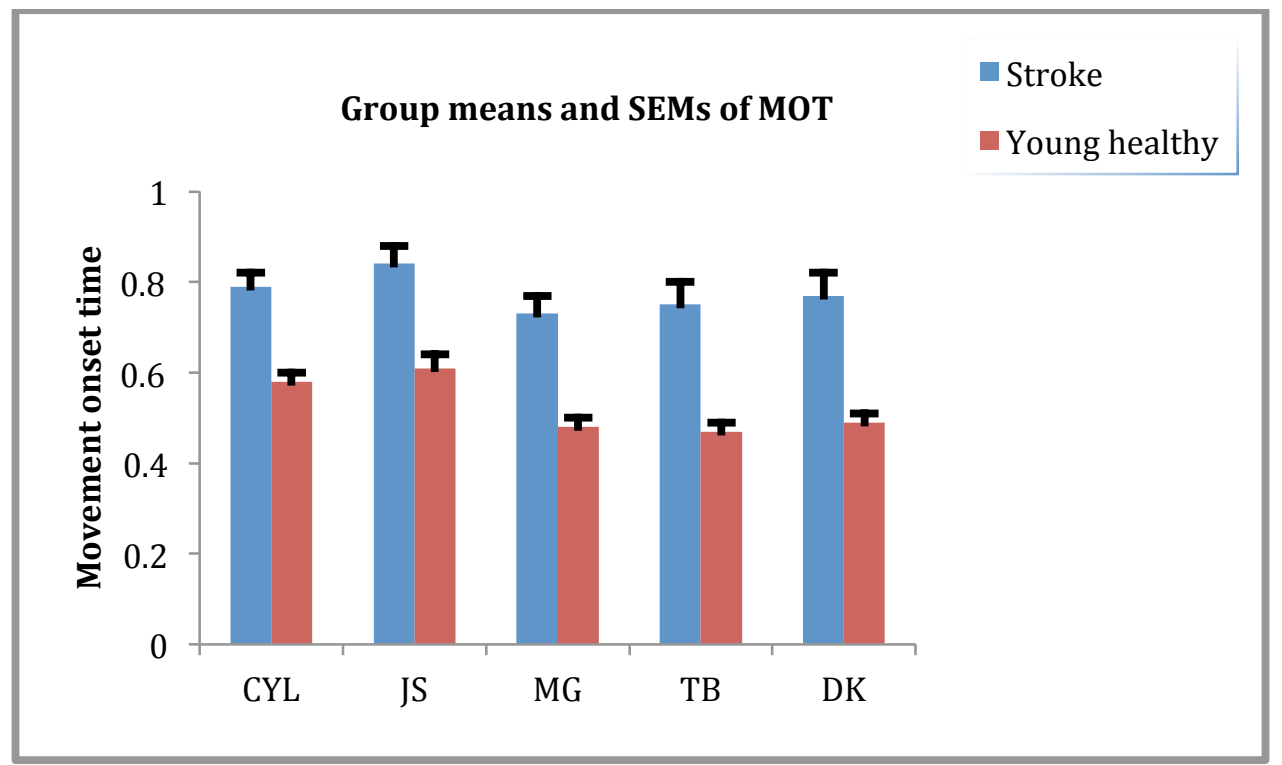

Figure 8- between group comparisons of MOT. Blue bars represent the performance of stroke group, and red bars represent the performance of the healthy group. On the $\mathrm{X}$-axis are the 5 objects that were manipulated during the tasks and on the $\mathrm{Y}$-axis is the MOT outcome measure in seconds. The error caps represent the standard error of the mean for both groups.

Table 9-between group statistical analyses of MOT

\begin{tabular}{|lll|}
\hline Movement onset & t statistics $(\mathbf{d f}=\mathbf{3 8})$ & Sig. (2-tailed) \\
CYL & 5.58 & .000 \\
JS & 4.52 & .000 \\
MG & 5.56 & .000 \\
TB & 5.21 & .000 \\
DK & 5.20 & .000 \\
\hline
\end{tabular}


Development of a Computerized assessment tool for hand-arm function after stroke: test-retest reliability and convergent validity

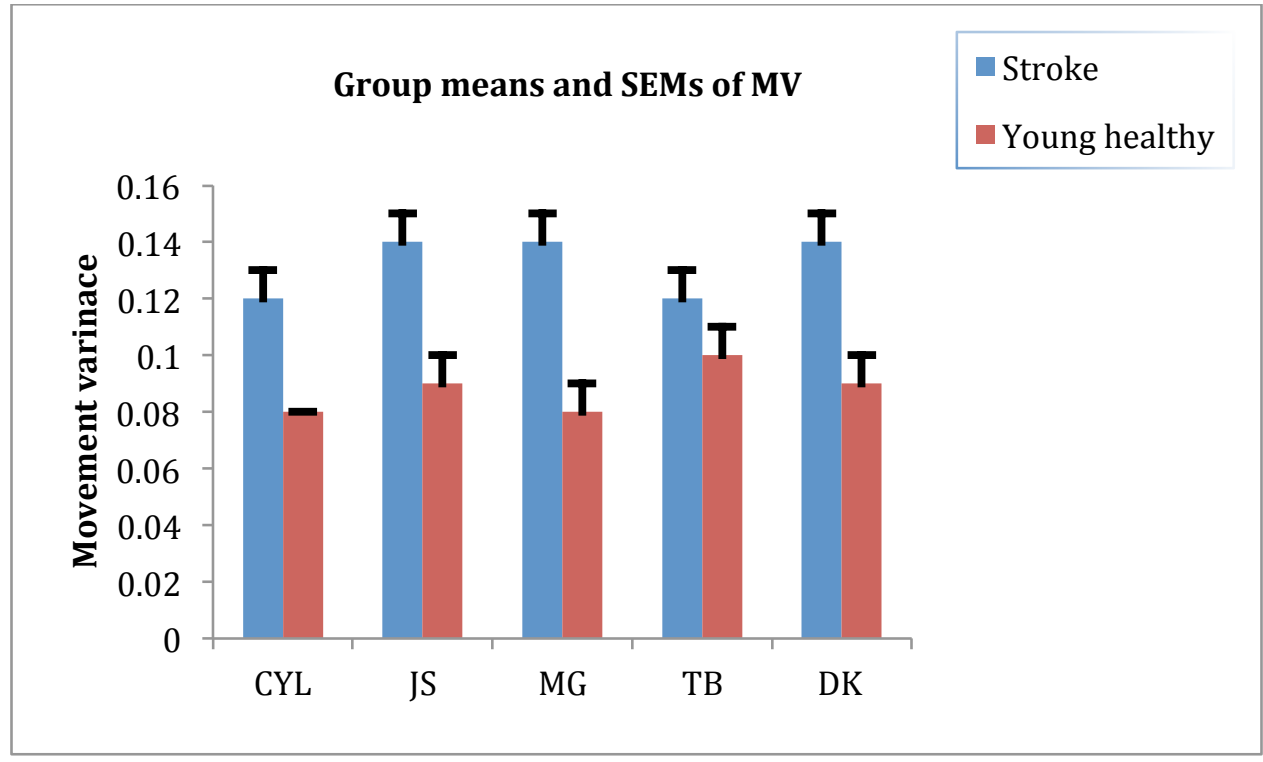

Figure 9-between group comparisons of MV. Blue bars represent the performance of stroke group, and red bars represent the performance of the healthy group. On the $\mathrm{X}$-axis are the 5 objects that were manipulated during the tasks and on the $\mathrm{Y}$ axis is the MV outcome measure. The error caps represent the standard error of the mean for both groups.

Table 10- between group statistical analyses of MV

\begin{tabular}{|lll|}
\hline Movement variance & t statistics $(\mathbf{d f}=\mathbf{3 8})$ & Sig. (2-tailed) \\
CYL & 3.31 & .002 \\
JS & 3.64 & .001 \\
MG & 5.21 & .000 \\
TB & 1.45 & .154 \\
DK & 3.23 & .003 \\
\hline
\end{tabular}


Development of a Computerized assessment tool for hand-arm function after stroke: test-retest reliability and convergent validity

\subsection{DISCUSSION}

This study had two major objectives. The first was to determine the test-retest reliability of the GHA assessment tool. Test-retest reliability ranged from high to moderate for TRE and AC performance measures of the Cyclic tracking mode and SR and MOT of the Motor skill game, whereas MV had moderate to low test-retest reliability.

The second objective was to determine the convergent validity of the GHA assessment. With very few exceptions there were no significant correlations between GHA and WMFT performance outcomes. Within stroke group correlation of GHA tasks revealed no or correlation. Further, a between-group comparison between stroke and healthy group to determine the known group validity of GHA performance measures was done. All the GHA Cyclic tracking and Motor skill game performance measures were significantly better in healthy than in stroke $(\mathrm{p}<0.05)$. 


\section{Development of a Computerized assessment tool for hand-arm function after stroke: test-retest reliability and convergent validity}

\subsubsection{Test-Retest Reliability}

For the 5 GHA performance measures (TRE and AC of the Cyclic tracking mode, $\mathrm{SR}, \mathrm{MOT}$ and MV of the motor skill game) the ICCs ranged from high to moderate for most of them. In general, the cyclic tracking performance measures (TRE and AC) had higher ICCs than the Motor skill game measures (MOT, SR, and MV). This could be explained by the fact that the Cyclic tracking tasks were comparatively easier to perform than the Motor skill game tasks. The Cyclic tracking tasks were predictable and continuous. Whereas, the Motor skill Game task had randomness, and required more accuracy and precision. Higher ICCs of TRE and AC indicate that participants were able to produce continuous movements but were not able to reciprocate the higher accuracy and precision demands of the Motor skill game tasks. For instance, ICCs were lowest for the MV of Motor skill game task, which could be due to poor accuracy and movement repeatability in a random task, pertaining to factors such as post-stroke spasticity and muscle weakness.

SEM and MDC values were low for the performance measures having high ICC values and were high for the performance measures having low ICC values. For instance, MV had the lowest ICCs and highest SEM and MDC values. This could be again explained by the poor movement repeatability, i.e. it was difficult to produce random and complex movement over and over than an easy and predictable movement. For daily activities, we are required to perform a movement not just once, but multiple times with high accuracy and precision. Thus, these findings suggest that post-stroke movement repeatability is poor. Spasticity, lack of range of motion, and movement control are other factors that could account for the same. Lastly, post-stroke compensatory movements are 


\section{Development of a Computerized assessment tool for hand-arm function after stroke: test-retest reliability and convergent validity}

seen, our tasks required participants to perform exact movements that would move the cursor on the screen and an associated movement would not move it to the same extent and thus movement error would increase.

Therefore, performance measures having high SEM and MDC such as MV would require a greater improvement for them to be significant when comparing the effects of a treatment program. In the case of MV movement repeatability with accuracy and precision has to be practiced more often.

ICC values ranged from high to low for different object-manipulation tasks. The test-retest findings interestingly reflected the complexity of a task, i.e. the more complex was the task lower was its ICC value and easier was the task, higher was the ICC value. A similar finding was observed for SEM and MDC values, i.e. more complex task had higher SEM and MDC values, whereas simpler task had lower SEM and MDC values.

The object manipulation tasks differ from each other in terms of functional demands, anatomical demands, and geometric properties. Object-manipulation having lower functional and anatomical demands had high ICCs for instance; doorknob rotation had a high to moderate ICC values in 4 of the 5 performance measures. The doorknob rotation requires small fine movements of the thumb and index finger, and a crafted object was used to eliminate object's mass and to restrict movement to a single axis of rotation. Thus, it was easy to manipulate than other objects. These could be the reasons for its best performance. Similar results were found when manipulating the jar lid. It had high to moderate ICC values. The movements required were very similar to that of the doorknob i.e. required grasp using the whole hand, and small movements of the thumb 


\section{Development of a Computerized assessment tool for hand-arm function after stroke: test-retest reliability and convergent validity}

and all the fingers with some forearm pronation and supination. Like doorknob, a crafted object was used to eliminate the object's mass and restrict movement to one axis.

Rolling the cylinder had high to moderate ICC values in most of the performance measures. Rolling of a soccer ball and rotating the joystick, on the contrary, had moderate to low ICCs. Manipulation of the cylinder, soccer ball, and joystick required movements of the wrist, elbow, and shoulder simultaneously. Finger extension was also required to handle the object while they were rotated. Though all three of them involved similar joints, their outcomes were different, which might be due to their geometric properties and functional demands. For instance objects with 2 or 3 degrees of freedom (joystick and soccer ball) could be manipulated in more than one plane and require high movement control, thus were difficult to handle. These objects had lower stability thus lower performance and lower ICC values. On the contrary objects with only 1 degree of freedom (rolling of a cylinder), was easy to handle as it only could be manipulated in one plane. Objects with 1 degree of freedom had higher stability thus better performance and higher ICC values.

Rotation of a beach ball was the bilateral task and required coordinated movements of both hands and arms (required ulnar-radial deviation of both wrists with the elbow in 90-degree flexion). Rotation of the mug required forearm pronation and supination. These were the two most difficult object manipulation tasks, with much lower ICCs. For bilateral and mug manipulation, controlled cyclic movements of involved joints were required. Poor coordination, the excessive tone in one hand and arm could attribute to these findings. Also, it was noticed that pronation and supination of the forearm are largely affected post stroke, and participants found the mug manipulation 
Development of a Computerized assessment tool for hand-arm function after stroke: test-retest reliability and convergent validity

task, in particular, the most challenging one. Thus, these findings could relate to the participant's perspective and results.

The object manipulation tasks with lower ICCs and higher SEM and MDC values indicate that higher performance measures of these tasks will be required to change for a significant difference than those with high ICC and lower SEM and DMCs. Thus, objectmanipulations having poor ICC, SEM and MDCs should be included in the participant's exercise program and practiced often.

Similar findings were reported in a previous study where test-retest reliability of similar performance and tasks were quantified in a population with hand arthritis. In this study, a task similar to the doorknob manipulation had high ICC (0.84), which required rotation of a wineglass using the thumb, index, and middle fingers. A task similar to the mug rotation had moderate ICC $(0.53)$, which required rotation of a jug. 
Development of a Computerized assessment tool for hand-arm function after stroke: test-retest reliability and convergent validity

The Cyclic tracking tasks had, in general, high ICC and lower SEM and MDC values than the Motor skill game tasks. Cylinder, soccer ball, mug and doorknob had high ICC and lower SEM and MDC for Cyclic tracking, whereas for the Motor skill game only jar lid and doorknob had high ICC. One of the reasons for these findings could be the randomness of the Motor skill game task, i.e. the targets appeared at random locations and required the client first to locate the target and then make movements of different amplitude. On the contrary, cyclic tracking was a continuous rhythmic task with fixed amplitude, which also made it predictable i.e. there was no random element present. Lower ICC values of the motor skill game could also be explained, as this task required greater movement precision (i.e. to interact with small moving targets). Also, the performance measures more directly quantify the movement accuracy and response time. Thus, the participants were able to perform relatively better during Cyclic tracking tasks as it was comparatively easy and predictable, but when it came to the Motor skill game tasks, due to high accuracy and precision demands, their performance level dropped down. 


\section{Development of a Computerized assessment tool for hand-arm function after stroke: test-retest reliability and convergent validity}

\subsubsection{Convergent validity and known group validity}

GHA performance measures for each object manipulation tasks were correlated with WMFT and grip strength to examine the convergent validity. In most of the cases, there were no significant correlations found between the GHA performance measures and WMFT or grip strength. Similar findings of low correlations were reported in a previous study (36) where task performance measures were correlated with a time-based outcome, nine hole peg test (NHPT) for hand arthritis participants. In this study, the participants were asked to place 9 pegs into 9 holes in a wooden block and then remove those pegs from the block, and the time was recorded. NHPT is similar to WMFT as it records the time taken to complete the test.

Likewise, the WMFT measures the time taken to complete a task, and also it rates movement quality on a 5-point ordinal scale. Though the WMFT tasks range from manipulation of gross to fine objects, time taken to complete a task does not tell us about movement quality, movement accuracy and response time. The GHA assessment tool, on the contrary, is designed to objectively measure movement quality, accuracy, amplitude and response time. Furthermore, the WMFT tasks required general object manipulation and did not require the precision of movement as that was required by the Motor skill game task of GHA. Also, WMFT tasks required limited movement repetition (although some tasks had more than one common object to manipulate), but the cyclic tracking task of GHA, on the other hand, required repetitive cyclic movements. Lastly, in the WMFT there are limited numbers of objects that are manipulated, whereas with GHA different kinds of object manipulation tasks could be done. These properties of GHA tool make it an objective assessment tool for upper extremity function. 


\section{Development of a Computerized assessment tool for hand-arm function after stroke: test-retest reliability and convergent validity}

A low correlation was also observed between GHA performance measures and grip strength. This finding again was not surprising as grip strength measures single voluntary maximum contraction of the wrist and hand muscles. Maximum voluntary contraction was not required during the object-manipulation of the GHA. Manipulation of the cylinder, soccer ball, and joystick required finger extension with controlled movements of the wrist, elbow and shoulder joints. The mug task did require a grasp to hold the handle, but it was a light grip and did not require maximum contraction of the wrist and hand muscles. For the jar lid and doorknob manipulation, a whole hand or twofinger grip was required, but these grips only required fingertips to hold the object. Lastly, for the bilateral task the participant held a beach ball and manipulated it by wrist ulnar-radial deviation. It is not the first time a low correlation is observed between task performances and grip strength; a previous study has reported similar findings (26).

A correlation of all GHA performance measures for all tasks within the stroke group was performed. There were no significant correlations among the different object manipulation tasks with one exception. The one exception was a high correlation for the TRE value between the rotation of the cylinder and soccer ball $(\mathrm{r}=0.72, \mathrm{p}<0.001)$. Although the cylinder was more stable than the soccer ball, the hand position, and wrist/elbow/shoulder motion was similar. Though the movements associated with the cylinder are shoulder, elbow flexion-extension and those with soccer ball are internal, external rotation of the shoulder and flexion-extension of the elbow, both of these tasks required finger and hand extension. Thus, the involvement of similar joints and similar movements might be the reason for their correlation. The insignificant correlations between the other performance measures for all tasks can be explained by the fact that all 


\section{Development of a Computerized assessment tool for hand-arm function after stroke: test-retest reliability and convergent validity}

the tasks were, in fact, different from each other regarding physical properties, ergonomic properties, and functional and anatomical demands. For instance, manipulation of the doorknob was completely different from that of the mug and the mug was different from the soccer ball and so were the rest of the tasks.

A between group comparison in stroke and healthy participants was done to determine the known group validity of the GHA performance measures. Results of the statistical analysis revealed that all the performance measures, (TRE, AC, SR, MOT and MV) were significantly better in the healthy group than in the stroke group $(p<0.05)$. From the perspective of the manifestation of stroke, all performance measures were affected by the stroke, some more than the others. This indicates that healthy subjects were able to perform the tasks with efficiency, accuracy, and precision irrespective of the high functional and anatomical demands of GHA tasks. Further, movement repeatability was better in the healthy subjects and thus had less movement variance than the stroke subjects. Variance was still seen in healthy subjects for tasks that required fine control (such as the mug task which required controlled movement of pronation and supination) still; it was significantly less than the stroke group. It was expected that the healthy group would perform better than the stroke group, and they did significantly. 


\section{Development of a Computerized assessment tool for hand-arm function after stroke: test-retest reliability and convergent validity}

\section{CHAPTER-3: CONCLUDING CHAPTER}

\subsection{CONCLUSION}

The goal of this study was to develop a home-based tele-rehabilitation system for hand-arm function post stroke with an embedded assessment tool. It is important to include a tele-monitoring module for home-based exercise programs to obtain timely feedback and monitor improvement in performance measures of how the client is progressing in their home program. The purpose of this study was to test the psychometric properties of the embedded assessment tool. Post-stroke a quality therapy program is important, but a detailed functional assessment tool is equally essential to assess, plan and improvise treatment paradigms. The GTR, on one hand, is a home based, highly engaging quality therapy platform; on the other hand, GHA is embedded in the GTR and is a computerized assessment tool for upper extremity functions. Psychometric properties of the GHA assessment tool were tested in this study for a stroke population. The GHA assessment tool had moderate to high test-retest reliability and low systematic errors in most of its performance measures, which makes it an accurate measure for upper extremity functional assessment i.e. it can repeatedly and reliably measure data of stroke clients. MV was one of the outcome measures having the highest values for MDC. Though it had the lowest performance, it might be the measure which improves the most i.e. it might be possible to see small pre to post changes in other performance measures such as SR, but larger changes could be seen for MV for similar amounts of treatment.

Further in this study, 7 common objects were chosen and tested to establish the test-retest reliability of the GHA assessment tool. These objects ranged from simple to complex in terms of size, shape, the degree of freedom, number of joints involved and 


\section{Development of a Computerized assessment tool for hand-arm function after stroke: test-retest reliability and convergent validity}

demands for accuracy and precision. These objects were in fact statistically different from each other. For example, an object was crafted that mimicked the function of a doorknob and another object was a typical soccer ball. The doorknob was very stable and required small movements of the thumb and index finger while the forearm were resting on firm foam. On the contrary, the soccer ball was a larger and more complex object and required multiple joints i.e. shoulder, elbow, wrist and hand for its manipulation. The soccer ball had multiple degrees of freedom. Thus it was difficult to manipulate. The participants had difficulty while placing their hand on the ball and manipulating it as it was very easy for the hand to come off the ball due to its multiple degrees of freedom. Objects that can be tested with the GHA tool are not limited, and different objects with different functional, anatomical and physiological demands can be tested with it. This wide range of objects and their evaluation is not available with any other assessment tool. Also, other assessment tools, such as the WMFT do not measure the quality of movement in terms of accuracy and precision. Thus the low correlation between the GHA performance measure and WMFT was not a surprise. This simply suggests that the two assessment tools represent distinct features or characteristics of the tasks performed. The GHA assessment tool measures the actual movement accuracy, variation and response time of a broad range of fine and gross motor task with different functional demands, whereas the WMFT measures the time taken to complete the tasks.

\subsection{CLINICAL SIGNIFCNACE}

So far the outcome measures for the upper extremity have focused on measuring the time of a task completion. Time is important but does not tell us about quality, efficiency, repetition, etc. of a movement. In real life, it is not the time that matters the 


\section{Development of a Computerized assessment tool for hand-arm function after stroke: test-retest reliability and convergent validity}

most but the movement quality and its repetition. It is important to identify the correct movement pattern and eliminate compensatory ones, which might be due to multiple reasons post stroke. Post-stroke movement patterns are different from a healthy person pertaining to lack of control and high muscle tone. Thus, it is important to educate the stroke client to perform movements like a healthy person and eliminate error to improve movement quality. Also, the hand and arm are the most mobile segments of our body and are engaged in almost every activity of daily living. Thus, assessing hand and arm functions by one or two object manipulations is not sufficient. Multiple sensory-motor functions are required to be evaluated before any treatment can be planned.

For this, we developed the GHA. The GHA is not a diagnostic tool but is developed to fill the above stated gaps in an assessment tool, and evaluate the difference between two treatment sessions in terms of movement accuracy, quality, success rate and variance. Also, the GHA helps to evaluate an individual's functional improvement and further requirements in a therapy program. The GHA is a part of the GTR and embedded in it. The GTR system uses commercially available computer games and object manipulation for physical therapy post-stroke. A thorough knowledge about objects and their geometrical properties, functional and anatomical demands, helps to set specific treatment goals for each individual. Transfer of the GTR to the participant's home is the ultimate goal, where the GHA will provide continuous feedback to the participant and therapist about their progress and prognosis.

\subsection{STRENGTHS AND LIMITATIONS}

The GHA assessment tool is a standardized program for analyzing movement and it's various components through the manipulation of a myriad of objects of daily living. 


\section{Development of a Computerized assessment tool for hand-arm function after stroke: test-retest reliability and convergent validity}

In the current study, a set of standardized objects was chosen to assess upper extremity functional status post stroke. The test-retest reliability and convergent validity of GHA was determined. The results of this study showed that the GHA is an objective assessment tool that can quantify the client's functional status, help in treatment planning, and compare changes between treatment sessions.

Unlike other assessment tools, the GHA quantifies a movement in terms of accuracy, quality, and success rate and movement variation. Other tools, on the contrary, measure the time taken to complete a task, or objects moved in a given time to complete a task. Time-based assessment tools are not able to quantify a movement like the GHA does. Also, the GHA is not limited to a certain number of objects; instead most of the objects of daily living can be manipulated and assessed, leaving a few such as playing a musical instrument. Playing an instrument requires very fine repetitive and random movements in more than one plane. The GHA can assess a movement at one time in one plane only.

The reliability of the GHA has been developed in the hand arthritis population and now in the stroke population. It is required to establish the reliability and validity of the GHA tool in other neurological populations such as traumatic brain injury and spinal cord injury.

The GHA has two modes that measure performance from different perspectives. Both the modes were simple and straightforward; interestingly both the modes did require some cognitive abilities, which were not dealt with before. People with previous knowledge of computers and games might be at some advantage, which is not listed in this study. Though every participant was given a detailed explanation of each task, 


\section{Development of a Computerized assessment tool for hand-arm function after stroke: test-retest reliability and convergent validity}

participants having experience with computers and games took less time to understand the tasks than the other participants. Lastly, tasks that required maximum contraction of hand and wrist muscles were not tested and correlated to grip strength in this study

\subsection{FUTURE DIRECTIONS}

As grip strength is an essential measure of hand assessment, manipulation tasks involving maximum muscle contraction should be tested and correlated with the same. Further, the reliability of GHA tool should be established in other populations with neurological conditions such as spinal cord injury, multiple sclerosis, and traumatic brain injury.

The GHA assessment tool is embedded in a GTR treatment program, that uses commercially available games and a large range of objects and utensils for everyday living for post-stroke rehabilitation, which increases compliance. The future goal is to conduct a feasibility study where treatment is provided with the GTR and participants are assessed multiple times with the GHA throughout the treatment program to observe and record the gradual recovery in stroke clients.

Most stroke patients clearly benefit from intensive inpatient and well-resourced outpatient care (4). While one-to-one supervised therapy is the preferred form for many patients, due to access and financial barriers many patients do not have this option (21). In fact, it is not unusual for the patient's physical function to decline after discharge from inpatient/outpatient rehabilitation in the home, when in terms of the natural course of their injury; they should be improving (40). When rehabilitation regimens are prescribed for the patients to do at home or community centers, adherence is low because 
Development of a Computerized assessment tool for hand-arm function after stroke: test-retest reliability and convergent validity

rehabilitation exercises are tedious and boring, and there is little or no support or followup (21).

To improve the adherence of physical therapy, an innovative program as been developed, the Game-based Tele-Rehabilitation program (GTR) of therapeutic exercises for hand and arm (65). The GTR consists of an innovative exercise gaming platform, through the novel use of computer input devices, which takes advantage of the wealth of inexpensive, easily accessible commercial computer games. This approach was designed to provide engaging, high-quality, client-centered therapy in the acute rehabilitation setting, and which could then be implemented in the home via tele-rehabilitation. At the core of the platform are designed rehabilitation games with automated monitoring that target fine and gross motor skills of the hand and arm (GHA). This not only provides an enhanced therapy program but also importantly allows automatic tracking of exercise duration and performance outcomes. The idea of a tool in which treatment and assessment are integrated is a very attractive system as it is efficient and time saving, provides measures of intensity, duration and compliance, and allows tracking of change in fine/gross motor skills within each treatment session and over time thus allowing examination of "dose-response" relationship. It also provides timely feedback and support for clients and clinicians. Electronic records and reports are also generated to inform therapists when and how a client is performing and how they are progressing in their individualized exercise program. This information will be valuable for discharge planning. Also, the development of a feasible and cost-effective program that can transition from the clinical setting to the home could allow early discharge. 


\section{Development of a Computerized assessment tool for hand-arm function after stroke: test-retest reliability and convergent validity}

Ten participants will be recruited in this study, and a task specific, exercise gaming program will be given. Each participant will be asked to attend 3-4 forty-five minute therapy sessions at HSC or RHC. These will be scheduled in the last couple of weeks of their inpatient stay when they have almost achieved their inpatient goals and are

close to discharge. A starting exercise program will be established based on the degree of their hemiparesis and functional status. Participants will receive training regarding the specific exercises, and the use of the motion mouse and computer games. To begin games will be selected with relatively slow and large target movements. Game speed/amplitude and precision will be progressed as tolerated. Based on the initial three clinical sessions, a home program will be prescribed and adapted to the participants' specific abilities and tolerance. Participants will be asked to perform their exercise programs 30 minutes per day, five days per week for 12 weeks. The research therapist will attend the participant's home to set-up the motion mouse and computer games and the exercise objects. The research therapist will phone the participants weekly to monitor progress, inquire about difficulties with the computer equipment, answer questions, and to progress exercises as outlined above.

The following primary outcome measures will be obtained:

1. Wolf motor function test.

2. Grip strength test using a dynamometer

3. The stroke impact scale, which is a stroke-specific self-report health status measure will be included in the assessment of stroke outcomes, including strength, hand function, 


\section{Development of a Computerized assessment tool for hand-arm function after stroke: test-retest reliability and convergent validity}

activities of daily living, mobility, communication, emotion, memory and thinking, and participation. This will be assessed in the home visit and immediately after completion of the 12-week home program.

Secondary outcome measures will include the GHA assessment as described above to quantify movement quality and movement accuracy. Assessments will be conducted before the start of the intervention and at the end of the 12-week home program.

Individual interviews will be conducted at the end of the 12 week home exercise program and a qualitative analysis will be performed to describe the feasibility and acceptability of the technologies, content, and delivery of the home exercise programs, personal and environmental factors that influenced doing the home exercise, and recommendations or modifications for improving the exercise programs. Feasible results from this study would direct us towards a powered sample size RCT. 


\section{REFERENCES}

(1) Di Carlo A. Human and economic burden of stroke. Age Ageing 2009 Jan;38(1):4-5.

(2) Kaur G, English C, Hillier S. How physically active are people with stroke in physiotherapy sessions aimed at improving motor function? A systematic review. Stroke research and treatment $2012 ; 2012$.

(3) Wolf SL, Winstein CJ, Miller JP, Taub E, Uswatte G, Morris D, et al. Effect of constraintinduced movement therapy on upper extremity function 3 to 9 months after stroke: the EXCITE randomized clinical trial. JAMA 2006;296(17):2095-2104.

(4) Bassett SF. The assessment of patient adherence to physiotherapy rehabilitation. New Zealand journal of physiotherapy 2003;31(2):60-66.

(5) Roger VL, Go AS, Lloyd-Jones DM, Benjamin EJ, Berry JD, Borden WB, et al. Heart disease and stroke statistics--2012 update: a report from the American Heart Association. Circulation 2012 Jan 3;125(1):e2-e220.

(6) Mutai H, Furukawa T, Araki K, Misawa K, Hanihara T. Long - term outcome in stroke survivors after discharge from a convalescent rehabilitation ward. Psychiatry Clin Neurosci 2013;67(6):434-440.

(7) Nudo R. Recovery after brain injury: mechanisms and principles. Frontiers In Human Neuroscience; Front.Hum.Neurosci. 2013;7.

(8) Woldag H, Stupka K, Hummelsheim H. Repetitive training of complex hand and arm movements with shaping is beneficial for motor improvement in patients after stroke. J Rehabil Med 2010;42(6):582-587.

(9) Morris DM, Uswatte G, Crago JE, Cook EW, Taub E. The reliability of the Wolf Motor Function Test for assessing upper extremity function after stroke. Arch Phys Med Rehabil 2001;82(6):750-755.

(10) Uswatte G, Taub E, Morris D, Vignolo M, McCulloch K. Reliability and validity of the upper-extremity Motor Activity Log-14 for measuring real-world arm use. Stroke 2005 Nov;36(11):2493-2496.

(11) Narayan Arya K, Verma R, Garg R, Sharma V, Agarwal M, Aggarwal G. Meaningful taskspecific training (MTST) for stroke rehabilitation: a randomized controlled trial. Topics in stroke rehabilitation 2012;19(3):193-211. 


\section{Development of a Computerized assessment tool for hand-arm function after stroke: test- retest reliability and convergent validity}

(12) Sullivan KJ, Tilson JK, Cen SY, Rose DK, Hershberg J, Correa A, et al. Fugl-Meyer assessment of sensorimotor function after stroke: standardized training procedure for clinical practice and clinical trials. Stroke 2011 Feb;42(2):427-432.

(13) Van der Lee, Johanna H, de Groot V, Beckerman H, Wagenaar RC, Lankhorst GJ, Bouter LM. The intra-and interrater reliability of the action research arm test: a practical test of upper extremity function in patients with stroke. Arch Phys Med Rehabil 2001;82(1):14-19.

(14) Kwakkel G, Rietberg M, van Wegen E. Constraint-induced movement therapy improves upper extremity motor function after stroke. Australian Journal of Physiotherapy 2007;53(2):132.

(15) Azab M, Al-Jarrah M, Nazzal M, Maayah M, Abu Sammour M, Jamous M. Effectiveness of constraint-induced movement therapy (CIMT) as home-based therapy on Barthel Index in patients with chronic stroke. Topics in stroke rehabilitation 2009;16(3):207-211.

(16) Della Pietra GL, Savio K, Oddone E, Reggiani M, Monaco F, Leone MA. Validity and reliability of the Barthel index administered by telephone. Stroke 2011 Jul;42(7):2077-2079.

(17) Miltner WH, Bauder H, Sommer M, Dettmers C, Taub E. Effects of constraint-induced movement therapy on patients with chronic motor deficits after stroke: a replication. Stroke 1999 Mar;30(3):586-592.

(18) Page SJ, Gater DR, Bach-y-Rita P. Reconsidering the motor recovery plateau in stroke rehabilitation. Arch Phys Med Rehabil 2004;85(8):1377-1381.

(19) Dahl AE, Askim T, Stock R, Langorgen E, Lydersen S, Indredavik B. Short- and long-term outcome of constraint-induced movement therapy after stroke: a randomized controlled feasibility trial. Clin Rehabil 2008 May;22(5):436-447.

(20) Dromerick AW, Edwards DF, Hahn M. Does the application of constraint-induced movement therapy during acute rehabilitation reduce arm impairment after ischemic stroke? Stroke 2000;31(12):2984-2988.

(21) Clay DL, Hopps JA. Treatment adherence in rehabilitation: The role of treatment accommodation. Rehabilitation Psychology 2003;48(3):215.

(22) Rizzo A, Kim GJ. A SWOT analysis of the field of virtual reality rehabilitation and therapy. Presence 2005;14(2):119-146.

(23) Kwakkel G, Kollen BJ, Wagenaar RC. Therapy impact on functional recovery in stroke rehabilitation: a critical review of the literature. Physiotherapy 1999;85(7):377-391.

(24) Kwakkel G, van Peppen R, Wagenaar RC, Wood Dauphinee S, Richards C, Ashburn A, et al. Effects of augmented exercise therapy time after stroke: a meta-analysis. Stroke 2004 Nov;35(11):2529-2539. 


\section{Development of a Computerized assessment tool for hand-arm function after stroke: test- retest reliability and convergent validity}

(25) Mathers CD, Loncar D. Projections of global mortality and burden of disease from 2002 to 2030. Plos med 2006;3(11):e442.

(26) Kalra L. Stroke rehabilitation 2009: old chestnuts and new insights. Stroke 2010 Feb;41(2):e88-90.

(27) Laver K, George S, Thomas S, Deutsch J, Crotty M. VR for stroke rehabilitation. Cochrane Database Syst Rev 2011;7(9).

(28) Hijmans JM, James LA. Bilateral upper-limb rehabilitation after stroke using a movementbased game controller. Journal of rehabilitation research and development 2011;48(8):1005.

(29) Saposnik G, Mamdani M, Bayley M, Thorpe K, Hall J, Cohen L, et al. Effectiveness of Virtual Reality Exercises in STroke Rehabilitation (EVREST): rationale, design, and protocol of a pilot randomized clinical trial assessing the Wii gaming system. International Journal of Stroke 2010;5(1):47-51.

(30) Desrosiers J, Bravo G, Hebert R, Dutil E, Mercier L. Validation of the Box and Block Test as a measure of dexterity of elderly people: reliability, validity, and norms studies. Arch Phys Med Rehabil 1994 Jul;75(7):751-755.

(31) Atroshi I, Gummesson C, Andersson B, Dahlgren E, Johansson A. The disabilities of the arm, shoulder and hand (DASH) outcome questionnaire: reliability and validity of the Swedish version evaluated in 176 patients. Acta Orthop Scand 2000;71(6):613-618.

(32) Sin H, Lee G. Additional virtual reality training using Xbox Kinect in stroke survivors with hemiplegia. Am J Phys Med Rehabil 2013 Oct;92(10):871-880.

(33) da Silva Cameirão M, Bermúdez i Badia S, Duarte E, Verschure PF. Virtual reality based rehabilitation speeds up functional recovery of the upper extremities after stroke: a randomized controlled pilot study in the acute phase of stroke using the rehabilitation gaming system. Restorative Neurol Neurosci 2011;29(5):287.

(34) Standen PJ, Threapleton K, Connell L, Richardson A, Brown DJ, Battersby S, et al. Patients' use of a home-based virtual reality system to provide rehabilitation of the upper limb following stroke. Phys Ther 2015 Mar;95(3):350-359.

(35) A feasibility study of an upper limb rehabilitation system using kinect and computer games. Engineering in Medicine and Biology Society (EMBC), 2012 Annual International Conference of the IEEE: IEEE; 2012.

(36) Srikesavan CS, Shay B, Szturm T. Test-Retest Reliability and Convergent Validity of a Computer Based Hand Function Test Protocol in People with Arthritis. The open orthopaedics journal 2015;9:57. 


\section{Development of a Computerized assessment tool for hand-arm function after stroke: test- retest reliability and convergent validity}

(37) Shaughnessy M, Resnick BM, Macko RF. Testing a model of post- stroke exercise behavior. Rehabilitation nursing : the official journal of the Association of Rehabilitation Nurses 2006;31(1):15.

(38) Baran M, Lehrer N, Duff M, Venkataraman V, Turaga P, Ingalls T, et al. Interdisciplinary concepts for design and implementation of mixed reality interactive neurorehabilitation systems for stroke. Phys Ther 2015 Mar;95(3):449-460.

(39) Hammond ERA, Shay BL, Szturm T. Objective evaluation of fine motor manipulation-A new clinical tool. Journal of Hand Therapy 2009;22(1):28-36.

(40) Hendricks HT, van Limbeek J, Geurts AC, Zwarts MJ. Motor recovery after stroke: a systematic review of the literature. Arch Phys Med Rehabil 2002;83(11):1629-1637.

(41) Russell TG. Physical rehabilitation using telemedicine. J Telemed Telecare 2007;13(5):217220.

(42) Winters JM. Telerehabilitation research: emerging opportunities. Annu Rev Biomed Eng 2002;4(1):287-320.

(43) Granger CV. The emerging science of functional assessment: our tool for outcomes analysis. Arch Phys Med Rehabil 1998;79(3):235-240.

(44) Wolf SL, Catlin PA, Ellis M, Archer AL, Morgan B, Piacentino A. Assessing Wolf motor function test as outcome measure for research in patients after stroke. Stroke 2001;32(7):16351639.

(45) Desrosiers J, Bravo G, Hebert R, Dutil E, Mercier L. Validation of the Box and Block Test as a measure of dexterity of elderly people: reliability, validity, and norms studies. Arch Phys Med Rehabil 1994 Jul;75(7):751-755.

(46) Platz T, Pinkowski C, van Wijck F, Kim IH, di Bella P, Johnson G. Reliability and validity of arm function assessment with standardized guidelines for the Fugl-Meyer Test, Action Research Arm Test and Box and Block Test: a multicentre study. Clin Rehabil 2005 Jun;19(4):404-411.

(47) Duncan PW, Propst M, Nelson SG. Reliability of the Fugl-Meyer assessment of sensorimotor recovery following cerebrovascular accident. Phys Ther 1983 Oct;63(10):16061610 .

(48) Chen $\mathrm{H}$, Lin $\mathrm{K}, \mathrm{Wu} \mathrm{C}$, Chen $\mathrm{C}$. Rasch validation and predictive validity of the action research arm test in patients receiving stroke rehabilitation. Arch Phys Med Rehabil 2012;93(6):1039-1045. 


\section{Development of a Computerized assessment tool for hand-arm function after stroke: test- retest reliability and convergent validity}

(49) Uswatte G, Taub E, Morris D, Vignolo M, McCulloch K. Reliability and validity of the upper-extremity Motor Activity Log-14 for measuring real-world arm use. Stroke 2005 Nov;36(11):2493-2496.

(50) Uswatte G, Taub E, Morris D, Light K, Thompson PA. The Motor Activity Log-28: assessing daily use of the hemiparetic arm after stroke. Neurology 2006 Oct 10;67(7):1189-1194.

(51) Gummesson C, Atroshi I, Ekdahl C. The disabilities of the arm, shoulder and hand (DASH) outcome questionnaire: longitudinal construct validity and measuring self-rated health change after surgery. BMC musculoskeletal disorders 2003;4(1):1.

(52) Beaton DE, Katz JN, Fossel AH, Wright JG, Tarasuk V, Bombardier C. Measuring the wole or the parts?: Validity, reliability, and responsiveness of the disabilities of the arm, shoulder and hand outcome measure in different regions of the upper extremity. Journal of Hand Therapy 2001;14(2):128-142.

(53) Andersen Hammond ER, Shay BL, Szturm T. Objective evaluation of fine motor manipulation-a new clinical tool. J Hand Ther 2009 Jan-Mar;22(1):28-35; quiz 36.

(54) Lockery D, Peters JF, Ramanna S, Shay BL, Szturm T. Store-and-feedforward adaptive gaming system for hand-finger motion Cyclic tracking in telerehabilitation. IEEE Transactions on Information Technology in Biomedicine 2011;15(3):467-473.

(55) Szturm T, Maharjan P, Marotta JJ, Shay B, Shrestha S, Sakhalkar V. The interacting effect of cognitive and motor task demands on performance of gait, balance and cognition in young adults. Gait Posture 2013;38(4):596-602.

(56) Szturm T, Sakhalkar V, Boreskie S, Marotta JJ, Wu C, Kanitkar A. Integrated testing of standing balance and cognition: Test-retest reliability and construct validity. Gait Posture 2015;41(1):146-152.

(57) Bartko JJ. The intraclass correlation coefficient as a measure of reliability. Psychol Rep 1966 Aug;19(1):3-11.

(58) Koch GG. Intraclass correlation coefficient. Encyclopedia of statistical sciences 1982.

(59) Rankin G, Stokes M. Reliability of assessment tools in rehabilitation: an illustration of appropriate statistical analyses. Clin Rehabil 1998 Jun;12(3):187-199.

(60) Lexell JE, Downham DY. How to assess the reliability of measurements in rehabilitation. Am J Phys Med Rehabil 2005 Sep;84(9):719-723.

(61) Harvill LM. Standard error of measurement. Educational Measurement: issues and practice $1991 ; 10(2): 33-41$. 
Development of a Computerized assessment tool for hand-arm function after stroke: testretest reliability and convergent validity

(62) Lin KC, Hsieh YW, Wu CY, Chen CL, Jang Y, Liu JS. Minimal detectable change and clinically important difference of the Wolf Motor Function Test in stroke patients. Neurorehabil Neural Repair 2009 Jun;23(5):429-434.

(63) Fritz SL, Blanton S, Uswatte G, Taub E, Wolf SL. Minimal detectable change scores for the Wolf Motor Function Test. Neurorehabil Neural Repair 2009 Sep;23(7):662-667.

(64) Nijland R, van Wegen E, Verbunt J, van Wijk R, van Kordelaar J, Kwakkel G. A comparison of two validated tests for upper limb function after stroke: The Wolf Motor Function Test and the Action Research Arm Test. J Rehabil Med 2010;42(7):694-696.

(65) Srikesavan CS, Shay B, Robinson DB, Szturm T. Task-oriented training with computer gaming in people with rheumatoid arthritisor osteoarthritis of the hand: study protocol of a randomized controlled pilot trial. Trials 2013 Mar 9;14:69-6215-14-69. 